\title{
Local theory for 2-functors on path 2-groupoids
}

\author{
Urs Schreiber $^{1}$ - Konrad Waldorf ${ }^{2}$
}

Received: 27 November 2013 / Accepted: 29 June 2016 / Published online: 23 July 2016

(C) Tbilisi Centre for Mathematical Sciences 2016

\begin{abstract}
This article is concerned with 2-functors defined on the path 2-groupoid of a smooth manifold. We set up a procedure to extract local data of such 2-functors, similar to the extraction of transition functions of a fibre bundle. The main result of this paper establishes an equivalence between the globally defined 2-functors and their local data. This is a contribution to a project that provides an axiomatic formulation of connections on (possibly non-abelian) gerbes in terms of 2-functors, of which the present paper provides the first part. The second part provides equivalences between the local data, on one side, and various existing versions of gerbes with connection on the other side.
\end{abstract}

Keywords Smooth paths · Fundamental 2-groupoid · Descent theory · Parallel transport · Non-abelian gerbes

Mathematics Subject Classification Primary 18D05; Secondary 55R65 - 18D05

Communicated by Jim Stasheff.

$\bowtie \quad$ Urs Schreiber

urs.schreiber@gmail.com

Konrad Waldorf

konrad.waldorf@mathematik.uni-regensburg.de

1 Institute of Mathematics, Czech Academy of Sciences, Zitna 25, 11567 Praha 1, Prague, Czech Republic

2 Fakultät für Mathematik, Universität Regensburg, Universitätsstraß e 31, 93053 Regensburg, Germany 


\section{Contents}

1 Introduction . . . . . . . . . . . . . . . . . . . . . . . . 618

2 Locally trivial 2 -functors and their descent data . . . . . . . . . . . . . . . . 621

2.1 Local trivializations . . . . . . . . . . . . . . . . . . . . . . . . . 621

2.2 Descent data . . . . . . . . . . . . . . . . . . . . . . . . . . 622

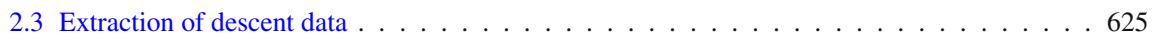

3 Reconstruction from descent data . . . . . . . . . . . . . . . . . . . 628

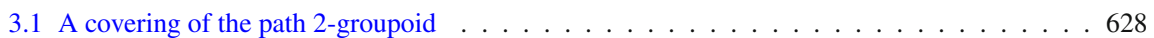

3.2 The Section 2-Functor . . . . . . . . . . . . . . . . . . . . . . 631

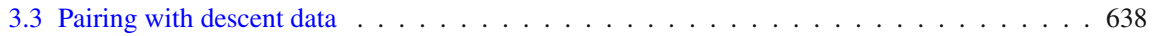

4 Local: global equivalence . . . . . . . . . . . . . . . . . . . . . . . . . . 644

4.1 Equivalence for a fixed cover . . . . . . . . . . . . . . . . . . . . . 644

4.2 Equivalence in the direct limit $\ldots \ldots \ldots$. . . . . . . . . . . . . . . 647

Appendix A: Basic 2-category theory . . . . . . . . . . . . . . . . . . . . . . . . . . . . . 649

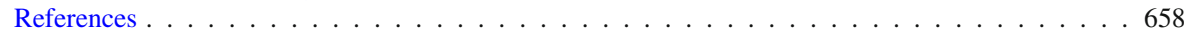

\section{Introduction}

The present article is a contribution to the axiomatic formulation of connections on (possibly non-abelian) gerbes carried out by the authors in four articles: the present one and [4-6]. The article [6] contains the main result of this project, and relies on the results of the other three articles.

The axiomatic formulation of connections on gerbes is based on 2-functors

$$
F: \mathcal{P}_{2}(M) \rightarrow T
$$

defined on the path 2-groupoid of a smooth manifold $M$, with values in some "target" 2-category $T$. The path 2-groupoid $\mathcal{P}_{2}(M)$ is a strict 2-groupoid with objects the points of $M, 1$-morphisms certain homotopy classes of paths, and 2-morphisms certain homotopy classes of homotopies between paths. A typical example of a target 2-category is the 2-category of algebras (over some fixed field), bimodules, and intertwiners. In that example, the algebra $F(x) \in T$ associated to a point $x \in M$ is supposed to be the fibre of the gerbe at $x$, the bimodule

$$
F(\gamma): F(x) \rightarrow F(y)
$$

associated to a path $\gamma$ from $x$ to $y$ is supposed to be the parallel transport of the connection on that gerbe along the path $\gamma$, and the intertwiner

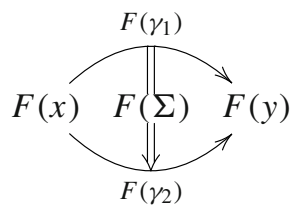

associated to a homotopy $\Sigma$ between paths $\gamma_{1}, \gamma_{2}$ from $x$ to $y$ is supposed to be the parallel transport of the connection on that gerbe along the surface $\Sigma$. The axioms 
of a 2-functor implement the "gluing laws" for parallel transport. The main difficulty in this approach to parallel transport of connections on gerbes is to distinguish those 2-functors which have the appropriate analytical behavior.

In this article we discuss local aspects of 2-functors defined on path 2-groupoids. Our first objective is to implement the notions of structure 2-groupoid, local trivializations, and descent data for a 2-functor F. Opposing connections on gerbes with connections on fibre bundles, these notions correspond, respectively, to the ones of typical fibre, local trivializations, and transition functions.

In Sect. 2 we set up the basis for our discussion of local properties. In Sect. 2.1 we introduce the notion of a local trivialization of a 2-functor $F: \mathcal{P}_{2}(M) \rightarrow T$ (Definition 2.1.1), which is central for this article. In order to say what a local trivializations is, we fix a strict 2-groupoid Gr and a 2-functor $i: \mathrm{Gr} \rightarrow T$, which together form a structure 2-groupoid for $F$. Basically, a 2-functor $F$ is locally $i$-trivializable if it factors locally through the 2-functor $i$. In more detail, we require that there exists a surjective submersion $\pi: Y \rightarrow M$, a strict 2-functor triv $: \mathcal{P}_{2}(Y) \rightarrow \mathrm{Gr}$, and a natural equivalence

$$
F \circ \pi_{*} \cong i \circ \text { triv, }
$$

where $\pi_{*}: \mathcal{P}_{2}(Y) \rightarrow \mathcal{P}_{2}(M)$ is the induced 2-functor on path 2-groupoids. Locally $i$-trivializable 2-functors form a 2-category $\operatorname{Funct}_{i}\left(\mathcal{P}_{2}(M), T\right)$.

In Sect. 2.2 we introduce the notion of descent data for locally trivialized 2-functors. The descent data of a local trivialization consists of the 2-functor

$$
\text { triv }: \mathcal{P}_{2}(Y) \rightarrow \mathrm{Gr}
$$

and further coherence data related to the natural equivalence (1.1). Descent data with respect to the structure 2-groupoid $i: \mathrm{Gr} \rightarrow T$ forms a 2-category $\mathfrak{D e s}^{2}(i)_{M}$. In Sect. 2.3 we describe how to extract descent data from a given local trivialization of a 2-functor $F$.

Descent data plays a crucial role in the before-mentioned process of distinguishing those 2-functors with the appropriate analytical behavior: it allows to impose smoothness conditions for the 2-functor $F$. We shall briefly outline these conditions - the full discussion is given in our concluding article [6]. We infer that the path 2-groupoid is a 2-groupoid internal to the category of diffeological spaces [5]. Diffeological spaces contain smooth manifolds as a full subcategory, but allow for many constructions that are in general not possible within the category of smooth manifolds. For example, the sets of 1-morphisms and 2-morphisms of the path 2-groupoid are quotients of subsets of mapping spaces between smooth manifolds, and all these operations lead to welldefined diffeological spaces. On the other side, however, the target 2-categories $T$ are typically not internal to the category of smooth manifolds or diffeological spaces, so that it is not possible to demand directly that $F$ is smooth. Instead, we assume that the structure 2-groupoid Gr is a Lie 2-groupoid, and then impose smoothness conditions for descent data of $F$, which is formulated with respect to Gr. For instance, the first of these smoothness conditions is that the 2-functor triv : $\mathcal{P}_{2}(Y) \rightarrow \mathrm{Gr}$ is smooth. There are further conditions related to the natural equivalence (1.1); these will be described in detail in [6]. 
In Sect. 3 we establish a procedure that reconstructs a 2 -functor from given descent data. This reconstruction procedure is, on a technical level, the main contribution of the present article. In order to explain some of the details, let $\mathfrak{D e s}_{\pi}^{2}(i) \subset \mathfrak{D e s}^{2}(i)_{M}$ denote the 2-category of descent data with respect to a fixed surjective submersion $\pi: Y \rightarrow M$. We introduce in Sect. 3.1 the codescent 2-groupoid $\mathcal{P}_{2}^{\pi}(M)$ whose idea is to combine the path 2-groupoid of $Y$ with additional "vertical jumps" in the fibres of $\pi$. The codescent 2-groupoid $\mathcal{P}_{2}^{\pi}(M)$ serves two purposes. Firstly, we prove in Sect. 3.2 the existence of a 2 -functor

$$
s: \mathcal{P}_{2}(M) \rightarrow \mathcal{P}_{2}^{\pi}(M)
$$

that consistently lifts points, paths, and homotopies between paths from $M$ to $Y$, by compensating the differences between local lifts with the vertical jumps. Secondly, we construct in Sect. 3.3 a "pairing 2-functor"

$$
R: \mathfrak{D e s}_{\pi}^{2}(i) \rightarrow \operatorname{Funct}\left(\mathcal{P}_{2}^{\pi}(M), T\right)
$$

expressing the result that the codescent 2 -groupoid $\mathcal{P}_{2}^{\pi}(M)$ is " $T$-dual" to the descent 2-category. The composition of a 2-functor in the image of $R$ with $s$ results in a locally $i$-trivializable 2 -functor on $\mathcal{P}_{2}(M)$, and this defines the reconstruction of a 2 -functor from descent data.

In Sect. 4 we prove the main result of this article, namely that the correspondence between globally defined locally $i$-trivializable 2-functors $F: \mathcal{P}_{2}(M) \rightarrow T$ and locally defined descent data is one-to-one. More precisely, we prove (Theorem 4.2.2) that extraction and reconstruction establish an equivalence

$$
\mathfrak{D e s}^{2}(i)_{M} \cong \operatorname{Funct}_{i}\left(\mathcal{P}_{2}(M), T\right)
$$

between the 2-category $\mathfrak{D e s}^{2}(i)_{M}$ of descent data with respect to the structure 2groupoid $i: \mathrm{Gr} \rightarrow T$ and the 2-category $\operatorname{Funct}_{i}\left(\mathcal{P}_{2}(M), T\right)$ of locally $i$-trivializable 2-functors.

In order to explain the importance of this result let us return to the anticipated discussion of smoothness conditions that we impose on the descent data. Let us denote by $\mathfrak{D e s}^{2}(i)_{M}^{\infty}$ the sub-2-category of $\mathfrak{D e s}^{2}(i)_{M}$ that consists of smooth descent data, which is defined in [6, Section 3]. The equivalence (1.2) from the main result of the present article restricts to an equivalence between $\mathfrak{D e s}^{2}(i)_{M}^{\infty}$ and a sub-2category of Funct ${ }_{i}\left(\mathcal{P}_{2}(M), T\right)$, called the 2-category of transport 2-functors on $M$ with $\mathrm{Gr}$-structure. These transport 2-functors constitute our axiomatic formulation of connections on gerbes. In this interpretation, our main theorem implies an equivalence between transport 2-functors and their smooth descent data. In several examples, described in [6, Section 4], smooth descent data has a clear geometrical description in terms of smooth functions and differential forms, and can be identified with existing versions of connections on gerbes. These identifications establish the relation between connections on gerbes and their parallel transport 2-functors.

We have included an appendix containing a brief summary of the notions and conventions from higher category theory that we use. 


\section{Locally trivial 2 -functors and their descent data}

In this section we introduce the central notions of the local theory of 2-functors.

\subsection{Local trivializations}

Let $M$ be a smooth manifold. For points $x, y \in M$, a path $\gamma: x \rightarrow y$ is a smooth map $\gamma:[0,1] \rightarrow M$ with $\gamma(0)=x$ and $\gamma(1)=y$. We require paths to have "sitting instants", i.e., to be locally constant around $\{0,1\}$. A bigon $\Sigma: \gamma \Rightarrow \gamma^{\prime}$ between two paths $\gamma, \gamma^{\prime}: x \rightarrow y$ is a smooth fixed-ends homotopy from $\gamma$ to $\gamma^{\prime}$ with sitting instants at $\gamma$ and $\gamma^{\prime}$. The path 2-groupoid $\mathcal{P}_{2}(M)$ [5] of a smooth manifold $M$ is a strict 2-groupoid with

(i) objects: the points of $M$.

(ii) 1-morphisms: rank-one homotopy classes of paths.

(iii) 2-morphisms: rank-two homotopy classes of bigons.

The process of taking classes by homotopies of certain rank is explained in [5]. For the purpose of this article, it suffices to accept that these assure the existence of strict, associative compositions and of strict inverses. For definitions and conventions related to 2-categories we refer to Appendix A. If $f: M \rightarrow N$ is a smooth map, we get a 2functor $f_{*}: \mathcal{P}_{2}(M) \rightarrow \mathcal{P}_{2}(N)$. For composable smooth maps $f$ and $g: N \rightarrow O$ we get

$$
(g \circ f)_{*}=g_{*} \circ f_{*} .
$$

In this article, we study 2 -functors

$$
F: \mathcal{P}_{2}(M) \rightarrow T
$$

for $T$ some 2-category called target 2-category. The 2-category of 2-functors (2.1.2) is denoted Funct $\left(\mathcal{P}_{2}(M), T\right)$. If $f: M \rightarrow N$ is a smooth map, the composition of a 2-functor $F: \mathcal{P}_{2}(N) \rightarrow T$ with the 2-functor $f_{*}: \mathcal{P}_{2}(M) \rightarrow \mathcal{P}_{2}(N)$ is denoted by

$$
f^{*} F:=F \circ f_{*}: \mathcal{P}_{2}(M) \rightarrow T .
$$

Local trivializations of a 2-functor $F: \mathcal{P}_{2}(M) \rightarrow T$ have three attributes:

(i) A strict 2-groupoid Gr, the structure 2-groupoid.

(ii) A 2-functor $i: \mathrm{Gr} \rightarrow T$ that indicates how the structure 2-groupoid is realized in the target 2-category.

(iii) A surjective submersion $\pi: Y \rightarrow M$ implementing locality.

For a surjective submersion $\pi: Y \rightarrow M$ the fibre products $Y^{[k]}:=Y \times_{M} \cdots \times{ }_{M} Y$ are again smooth manifolds, and the canonical projections $\pi_{i_{1} \ldots i_{p}}: Y^{[k]} \rightarrow Y^{[p]}$ to the indexed factors are smooth maps.

Definition 2.1.1 A $\pi$-local $i$-trivialization of a 2-functor

$$
F: \mathcal{P}_{2}(M) \rightarrow T
$$


is a pair (triv, $t$ ) of a strict 2-functor triv : $\mathcal{P}_{2}(Y) \rightarrow \mathrm{Gr}$ and of a pseudonatural equivalence

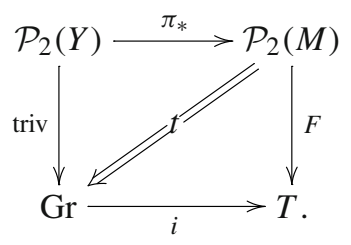

In other words, a 2-functor $F$ is locally trivializable, if its pullback $\pi^{*} F$ to the covering space factorizes - up to pseudonatural equivalence - through the structure 2groupoid $\mathrm{Gr}$. A 2-functor is called $i$-trivializable, if it has a id ${ }_{M}$-local $i$-trivialization. A 2-functor triv : $\mathcal{P}_{2}(M) \rightarrow$ Gr is called $i$-trivial, in which case we write $\operatorname{triv}_{i}:=i$ otriv in order to abbreviate the notation. We also remark that by "pseudonatural equivalence" we mean a pseudonatural transformation together with a weak inverse and two invertible modifications expressing the invertibility (see Appendix A).

We define a 2-category $\operatorname{Triv}_{\pi}^{2}(i)$ of 2-functors with $\pi$-local $i$-trivialization: an object is a triple $(F$, triv, $t)$ of a 2 -functor $F: \mathcal{P}_{2}(M) \rightarrow T$ together with a $\pi$-local $i$ trivialization (triv, $t$ ). A 1-morphism

$$
(F, \text { triv, } t) \rightarrow\left(F^{\prime}, \operatorname{triv}^{\prime}, t^{\prime}\right)
$$

is just a pseudonatural transformation $F \rightarrow F^{\prime}$ between the two 2-functors (ignoring the trivialization), and a 2-morphism is just a modification between those.

\subsection{Descent data}

Let $i: \mathrm{Gr} \rightarrow T$ be a 2-functor from a strict 2-groupoid Gr to a 2-category $T$, and let $\pi: Y \rightarrow M$ be a surjective submersion. In the following three definitions, we define a 2-category $\mathfrak{D e s}_{\pi}^{2}(i)$ of descent data.

Definition 2.2.1 A descent object is a tuple (triv, $g, \psi, f$ ) consisting of

(i) A strict 2-functor triv : $\mathcal{P}_{2}(Y) \rightarrow \mathrm{Gr}$

(ii) A pseudonatural equivalence $g: \pi_{1}^{*} \operatorname{triv}_{i} \rightarrow \pi_{2}^{*} \operatorname{triv}_{i}$

(iii) An invertible modification $\psi: \operatorname{id}_{\text {triv }_{i}} \Rightarrow \Delta^{*} g$

(iv) An invertible modification $f: \pi_{23}^{*} g \circ \pi_{12}^{*} g \Rightarrow \pi_{13}^{*} g$

such that the diagrams
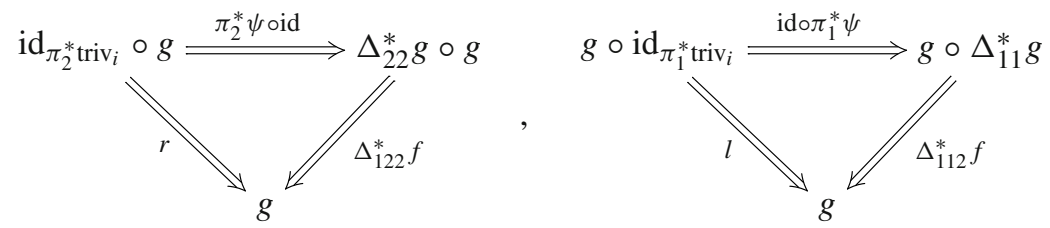
and

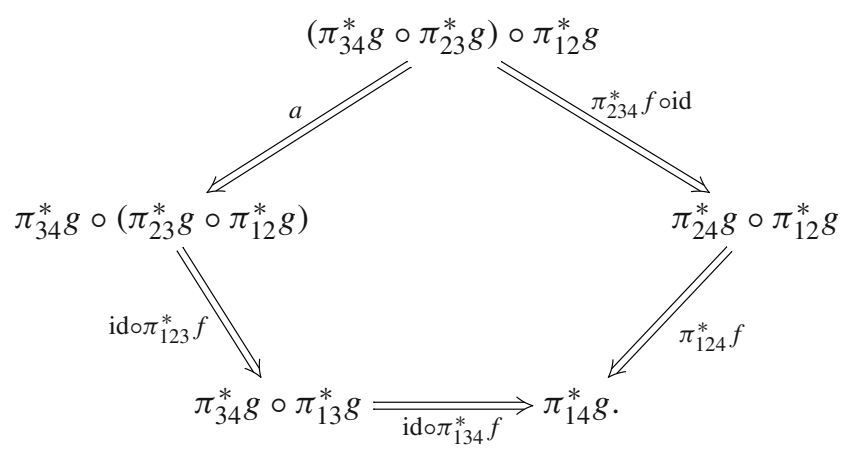

are commutative. A descent object (triv, $g, \psi, f$ ) is called normalized, if the conditions

$$
\operatorname{id}_{\text {triv }_{i}}=\Delta^{*} g \quad \text { and } \quad g \circ \Delta_{21}^{*} g=\operatorname{id}_{\pi_{1}^{*} \operatorname{triv}_{i}}
$$

and

$$
\psi=\mathrm{id}_{\Delta^{*} g} \quad \text { and } \quad \Delta_{121}^{*} f=\mathrm{id}_{\Delta_{11}^{*} g}
$$

hold.

In these diagrams, $r, l$ and $a$ are the right and left unifiers and the associator of the 2category $T$. Further is $\Delta: Y \rightarrow Y^{[2]}$ the diagonal map, $\Delta_{112}, \Delta_{122}: Y^{[2]} \rightarrow Y^{[3]}$ are the maps that duplicate the first and the second factor, respectively, $\Delta_{j j}:=\Delta \circ \pi_{j}$, and $\Delta_{21}: Y^{[2]} \rightarrow Y^{[2]}$ exchanges the two components. Normalized descent objects play an important role in the discussion of surface holonomy; see Lemma 3.3.4 and [6, Section5].

Definition 2.2.2 Let (triv, $g, \psi, f$ ) and (triv', $g^{\prime}, \psi^{\prime}, f^{\prime}$ ) be descent objects. A descent 1-morphism (triv, $g, \psi, f) \rightarrow\left(\operatorname{triv}^{\prime}, g^{\prime}, \psi^{\prime}, f^{\prime}\right)$ is a pair $(h, \varepsilon)$ of a pseudonatural transformation

$$
h: \operatorname{triv}_{i} \rightarrow \operatorname{triv}_{i}^{\prime}
$$

and an invertible modification

$$
\epsilon: \pi_{2}^{*} h \circ g \Longrightarrow g^{\prime} \circ \pi_{1}^{*} h
$$


such that the diagrams

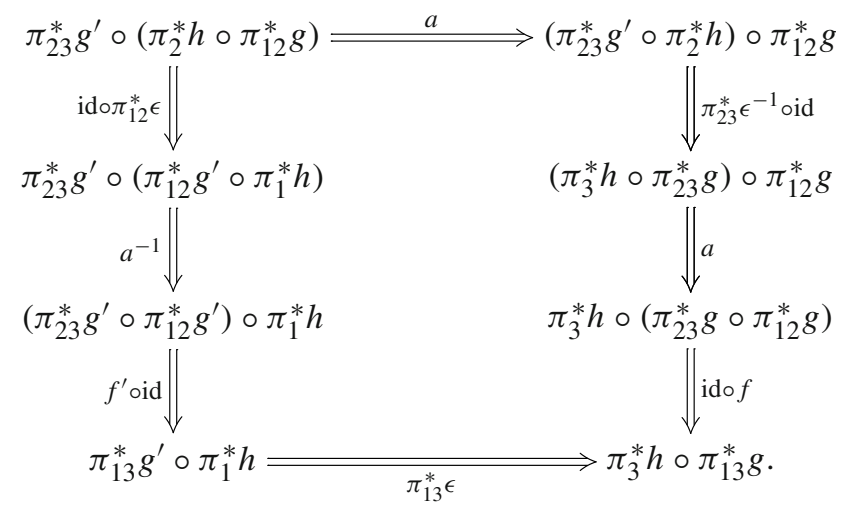

and

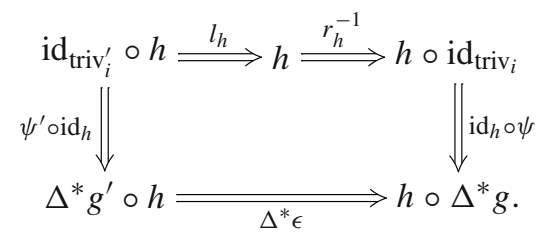

are commutative.

Finally, we introduce

Definition 2.2.3 Let $\left(h_{1}, \varepsilon_{1}\right)$ and $\left(h_{2}, \varepsilon_{2}\right)$ be descent 1-morphisms from a descent object (triv, $g, \psi, f$ ) to another descent object (triv', $\left.g^{\prime}, \psi^{\prime}, f^{\prime}\right)$. A descent 2morphism $\left(h_{1}, \varepsilon_{1}\right) \Rightarrow\left(h_{2}, \varepsilon_{2}\right)$ is a modification

$$
E: h_{1} \Rightarrow h_{2}
$$

such that the diagram

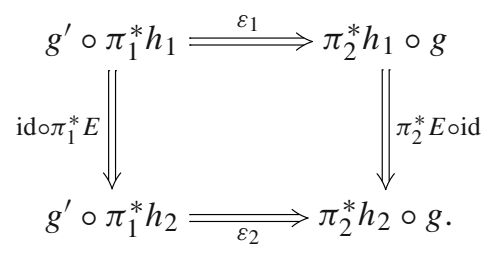

is commutative.

Descent objects, 1-morphisms and 2-morphisms form a 2-category $\mathfrak{D e s}_{\pi}^{2}(i)$ in an evident way. We remark that this 2-category comes with a strict 2-functor

$$
V: \mathfrak{D e s}_{\pi}^{2}(i) \rightarrow \operatorname{Funct}\left(\mathcal{P}_{2}(Y), T\right) .
$$


From a descent object (triv, $g, \psi, f$ ) it keeps only the 2-functor triv and from a descent 1-morphism $(h, \varepsilon)$ only the pseudonatural transformation $h$.

Example 2.2.4 We consider the case that the manifolds $M$ and $Y$ are just points. Let $\mathfrak{C}$ be a tensor category, and let $T:=\mathcal{B C}$ be the 2-category with one object associated to $\mathfrak{C}$, see Example A.2. Let $\mathrm{Gr}$ be the trivial 2-groupoid (one object, one 1-morphism and one 2-morphism), and let $i: \mathrm{Gr} \rightarrow T$ be the 2 -functor that sends the unique 1 -morphism to the tensor unit in $\mathfrak{C}$. Then, a descent object is precisely a special symmetric Frobenius algebra object in $\mathfrak{C}$.

Example 2.2.5 Let $\left\{U_{\alpha}\right\}_{\alpha \in A}$ be a cover of $M$ by open sets, $Y:=\bigsqcup_{\alpha \in A} U_{\alpha}$ the disjoint union of all open sets, and $\pi: Y \rightarrow M$ the projection, $\pi(\alpha, x):=x$. A descent object is then a collections of 2-functors $\operatorname{triv}_{\alpha}: P_{2}\left(U_{\alpha}\right) \rightarrow \mathrm{Gr}$, pseudonatural transformations $g_{\alpha \beta}: \operatorname{triv}_{\alpha} \rightarrow \operatorname{triv}_{\beta}$, and modifications $\psi_{\alpha}:$ id $\Rightarrow g_{\alpha \alpha}$ and $f_{\alpha \beta \gamma}: g_{\beta \gamma} \circ g_{\alpha \beta} \Rightarrow g_{\alpha \gamma}$. After imposing smoothness assumptions for descent data, as we describe in [6], this data can be expressed in differential-geometric language. Then, the 2-functor triv ${ }_{\alpha}$ turns out to be a certain 2-form on $U_{\alpha}$, the curving of the connection on the corresponding gerbe. The pseudonatural transformation $g_{\alpha \beta}$ turns out to be a 1-form on the double overlaps $U_{\alpha} \cap U_{\beta}$, which is sometimes called a connective structure. The modification $f_{\alpha \beta \gamma}$ turns out to be a smooth map on the triple overlaps $U_{\alpha} \cap U_{\beta} \cap U_{\gamma}$. Condition 2.2.2 of Definition 2.2.1 implies the Čech cocycle condition for $f_{\alpha \beta \gamma}$, finally defining a characteristic class of the underlying gerbe, living in $\mathrm{H}^{3}(M, \mathbb{Z})$.

\subsection{Extraction of descent data}

We have so far introduced a 2-category $\operatorname{Triv}_{\pi}^{2}(i)$ of 2-functors with $\pi$-local $i$-trivializations and a 2-category $\mathfrak{D e s}_{\pi}^{2}(i)$ of descent data, both associated to a surjective submersion $\pi$ and a 2-functor $i: \mathrm{Gr} \rightarrow T$. Now we define a 2 -functor

$$
\operatorname{Ex}_{\pi}: \operatorname{Triv}_{\pi}^{2}(i) \rightarrow \mathfrak{D e s}_{\pi}^{2}(i)
$$

between these 2-categories. This 2-functor extracts descent data from 2-functors with local trivializations.

Let $F: \mathcal{P}_{2}(M) \rightarrow T$ be a 2 -functor with a $\pi$-local $i$-trivialization (triv, $t$ ). We recall that by our conventions the pseudonatural equivalence $t$ comes with a weak inverse $\bar{t}: \operatorname{triv}_{i} \rightarrow \pi^{*} F$ and with invertible modifications

$$
i_{t}: \bar{t} \circ t \Rightarrow \operatorname{id}_{\pi^{*} F} \quad \text { and } \quad j_{t}: \operatorname{id}_{\text {triv }_{i}} \Rightarrow t \circ \bar{t}
$$

satisfying the identities (A.1). We define a pseudonatural equivalence

$$
g: \pi_{1}^{*} \operatorname{triv}_{\mathrm{i}} \rightarrow \pi_{2}^{*} \operatorname{triv}_{i}
$$

as the composition $g:=\pi_{2}^{*} t \circ \pi_{1}^{*} \bar{t}$ of pseudonatural equivalences. This composition is well-defined since $\pi_{1}^{*} \pi^{*} F=\pi_{2}^{*} \pi^{*} F$. We obtain $\Delta^{*} g=t \circ \bar{t}$, so that the definition $\psi:=j_{t}$ yields an invertible modification 


$$
\psi: \mathrm{id}_{\operatorname{triv}_{i}} \Rightarrow \Delta^{*} g
$$

Finally, we define an invertible modification

$$
f: \pi_{23}^{*} g \circ \pi_{12}^{*} g \Rightarrow \pi_{13}^{*} g
$$

as the composition

$$
\begin{aligned}
& \left(\pi_{3}^{*} t \circ \pi_{2}^{*} \bar{t}\right) \circ\left(\pi_{2}^{*} t \circ \pi_{1}^{*} \bar{t}\right) \Longrightarrow \pi_{3}^{*} t \circ\left(\left(\pi_{2}^{*} \bar{t} \circ \pi_{2}^{*} t\right) \circ \pi_{1}^{*} \bar{t}\right) \\
& \operatorname{ido}\left(\pi_{2}^{*} i_{t} \circ \mathrm{id}\right) \Downarrow \\
& \pi_{3}^{*} t \circ\left(\mathrm{id}_{\pi^{*} F} \circ \pi_{1}^{*} \bar{t}\right) \underset{\overline{\mathrm{idor} r_{1}^{*} \bar{t}}}{\Longrightarrow} \pi_{3}^{*} t \circ \pi_{1}^{*} \bar{t}
\end{aligned}
$$

where $r$ is the right unifier of $\operatorname{Funct}\left(\mathcal{P}_{2}\left(Y^{[2]}\right), T\right)$, and the first arrow summarizes two obvious occurrences of associators.

Lemma 2.3.1 The modifications $\psi$ and $f$ make the diagrams (2.2.1) and (2.2.2) commutative, so that

$$
\operatorname{Ex}_{\pi}(F, \operatorname{triv}, t):=(\operatorname{triv}, g, \psi, f)
$$

is a descent object.

Proof We prove the commutativity of the diagram on the left hand side of (2.2.1) by patching it together from commutative diagrams:

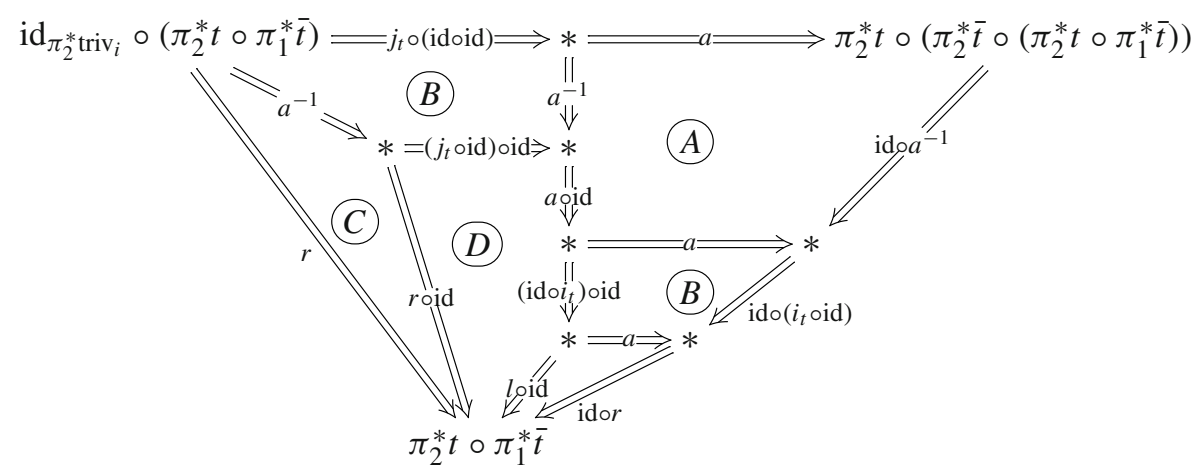

The six subdiagrams are commutative: A is the Pentagon axiom (C4) of $T$, B's are the naturality of the associator, $\mathrm{C}$ and $\mathrm{D}$ are diagrams that follow from the coherence theorem for the 2-category $T$, and the remaining small triangle is axiom (C2). The commutativity of the second diagram in (2.2.1) and the one of diagram (2.2.2) can be shown in the same way.

Now let $A: F \rightarrow F^{\prime}$ be a pseudonatural transformation between two 2-functors with $\pi$-local $i$-trivializations $t: \pi^{*} F \rightarrow \operatorname{triv}_{i}$ and $t^{\prime}: \pi^{*} F^{\prime} \rightarrow \operatorname{triv}_{i}^{\prime}$. Let $i_{t}, j_{t}$ 
and $i_{t^{\prime}}, j_{t^{\prime}}$ be the modifications (2.3.1) we have chosen for the weak inverses $\bar{t}$ and $\bar{t}^{\prime}$. We define a pseudonatural transformation

$$
h: \operatorname{triv}_{i} \rightarrow \operatorname{triv}_{i}^{\prime}
$$

by $h:=\left(t^{\prime} \circ \pi^{*} A\right) \circ \bar{t}$, and an invertible modification $\varepsilon$ by

$$
\begin{aligned}
& \pi_{2}^{*} h \circ g \Longrightarrow\left(\pi_{2}^{*} t^{\prime} \circ \pi_{2}^{*} \pi^{*} A\right) \circ\left(\left(\pi_{2}^{*} \bar{t} \circ \pi_{2}^{*} t\right) \circ \pi_{1}^{*} \bar{t}\right) \\
& \Downarrow\left(\pi_{2}^{*} l_{t^{\prime}}^{-1} \text { oid }\right) \circ\left(\pi_{2}^{*} i_{t} \circ \text { oid }\right) \\
& \left(\left(\pi_{2}^{*} t^{\prime} \circ \mathrm{id}\right) \circ \pi_{2}^{*} \pi^{*} A\right) \circ\left(\mathrm{id} \circ \pi_{1}^{*} \bar{t}\right) \\
& \Downarrow\left(\left(\text { ido } \pi_{1}^{*} i_{t^{\prime}}^{-1}\right) \circ \mathrm{id}\right) \circ \pi_{1}^{*} r_{t} \\
& \left(\left(\pi_{2}^{*} t^{\prime} \circ\left(\pi_{1}^{*} \bar{t}^{\prime} \circ \pi_{1}^{*} t^{\prime}\right)\right) \circ \pi_{1}^{*} \pi^{*} A\right) \circ \pi_{1}^{*} \bar{t} \Longrightarrow g^{\prime} \circ \pi_{1}^{*} h .
\end{aligned}
$$

Here, the unlabelled arrows summarize the definitions of $h$ and $g$ and several obvious occurrences of associators. Arguments similar to those given in the proof of Lemma 2.3.1 show the following lemma.

Lemma 2.3.2 The modification $\varepsilon$ makes the diagrams (2.2.3) and (2.2.4) commutative, so that $\operatorname{Ex}_{\pi}(A):=(h, \varepsilon)$ is a descent 1-morphism

$$
\operatorname{Ex}_{\pi}(A): \operatorname{Ex}_{\pi}(F) \rightarrow \operatorname{Ex}_{\pi}\left(F^{\prime}\right)
$$

In order to continue the definition of the 2-functor $\mathrm{Ex}_{\pi}$ we consider a modification $B: A_{1} \Rightarrow A_{2}$ between pseudonatural transformations $A_{1}, A_{2}: F \rightarrow F^{\prime}$ of 2-functors with $\pi$-local $i$-trivializations $t: \pi^{*} F \rightarrow \operatorname{triv}_{i}$ and $t^{\prime}: \pi^{*} F^{\prime} \rightarrow \operatorname{triv}_{i}^{\prime}$. Let $\left(h_{k}, \varepsilon_{k}\right):=\operatorname{Ex}_{\pi}\left(A_{k}\right)$ be the associated descent 1-morphisms for $k=1,2$. We define a modification $E: h_{1} \Rightarrow h_{2}$ by

$$
h_{1}=\left(t^{\prime} \circ \pi^{*} A_{1}\right) \circ \bar{t} \stackrel{\text { (ido } \left.\pi^{*} B\right) \circ \text { id }}{\longrightarrow}\left(t^{\prime} \circ \pi^{*} A_{2}\right) \circ \bar{t}=h_{2} .
$$

Lemma 2.3.3 The modification E makes the diagram (2.2.5) commutative so that $\operatorname{Ex}_{\pi}(B):=E$ is a descent 2-morphism

$$
\operatorname{Ex}_{\pi}(B): \operatorname{Ex}_{\pi}\left(A_{1}\right) \Rightarrow \operatorname{Ex}_{\pi}\left(A_{2}\right)
$$

In order to finish the definition of the 2-functor $\mathrm{Ex}_{\pi}$ we have to define its compositors and unitors. We consider two composable pseudonatural transformations $A_{1}: F \rightarrow F^{\prime}$ and $A_{2}: F^{\prime} \rightarrow F^{\prime \prime}$ and the extracted descent 1-morphisms $\left(h_{k}, \varepsilon_{k}\right):=\operatorname{Ex}_{\pi}\left(A_{k}\right)$ for $k=1,2$ and $(\tilde{h}, \tilde{\varepsilon}):=\operatorname{Ex}_{\pi}\left(A_{2} \circ A_{1}\right)$. The compositor

$$
c_{A_{1}, A_{2}}: \operatorname{Ex}_{\pi}\left(A_{2}\right) \circ \operatorname{Ex}_{\pi}\left(A_{2}\right) \Rightarrow \operatorname{Ex}_{\pi}\left(A_{2} \circ A_{1}\right)
$$


is the modification $h_{2} \circ h_{1} \Rightarrow \tilde{h}$ defined by

$$
\begin{aligned}
& \left(\left(t^{\prime \prime} \circ \pi^{*} A_{2}\right) \circ \bar{t}^{\prime}\right) \circ\left(\left(t^{\prime} \circ \pi^{*} A_{1}\right) \circ \bar{t}\right) \Longrightarrow\left(t^{\prime \prime} \circ\left(\pi^{*} A_{2} \circ\left(\left(\bar{t}^{\prime} \circ t^{\prime}\right) \circ \pi^{*} A_{1}\right)\right)\right) \circ \bar{t} \\
& \left.\Downarrow\left(\text { ido(ido }\left(i_{t^{\prime}} \circ \text { id }\right)\right)\right) \text { oid } \\
& \left(t^{\prime \prime} \circ\left(\pi^{*} A_{2} \circ\left(\operatorname{id} \circ \pi^{*} A_{1}\right)\right)\right) \circ \bar{t} \Longrightarrow\left(t^{\prime \prime} \circ \pi^{*}\left(A_{2} \circ A_{1}\right)\right) \circ \bar{t} .
\end{aligned}
$$

For a 2-functor $F: \mathcal{P}_{2}(M) \rightarrow T$ we find $\operatorname{Ex}_{\pi}\left(\mathrm{id}_{F}\right)=t \circ \bar{t}$. So, the unitor

$$
u_{F}: \operatorname{Ex}_{\pi}\left(\mathrm{id}_{F}\right) \Rightarrow \operatorname{id}_{\text {triv }_{i}}
$$

is the modification $u_{F}:=j_{t}^{-1}$. The identities (A.1) for $i_{t}$ and $j_{t}$ show that compositors and unitors are descent 2-morphisms. The following statement is now straightforward to check.

Proposition 2.3.4 The structure collected above furnishes a 2-functor

$$
\operatorname{Ex}_{\pi}: \operatorname{Triv}_{\pi}^{2}(i) \rightarrow \mathfrak{D e s}_{\pi}^{2}(i)
$$

\section{Reconstruction from descent data}

We have so far described how globally defined 2-functors induce locally defined structure, in terms of the 2-functor $\mathrm{Ex}_{\pi}$. In this section we describe a 2-functor going in the other direction.

\subsection{A covering of the path 2-groupoid}

In this section we introduce the codescent 2 -groupoid $\mathcal{P}_{2}^{\pi}(M)$ associated to a surjective submersion $\pi: Y \rightarrow M$. It combines the path 2-groupoid of $Y$ with additional jumps between the fibres. This construction generalizes the groupoid $\mathcal{P}_{1}^{\pi}(M)$ from [4].

The objects of $\mathcal{P}_{2}^{\pi}(M)$ are all points $a \in Y$. There are two "basic" 1-morphisms:

1. Paths: rank-one homotopy classes of paths $\gamma: a \rightarrow a^{\prime}$ in $Y$.

2. Jumps: points $\alpha \in Y^{[2]}$ considered as 1-morphisms from $\pi_{1}(\alpha)$ to $\pi_{2}(\alpha)$.

The set of 1-morphisms of $\mathcal{P}_{2}^{\pi}(M)$ is freely generated from these two basic 1morphisms, i.e. we have a formal composition $*$ and a formal identity $\mathrm{id}_{a}^{*}$ (the empty composition) associated to every object $a \in Y$. We introduce six "basic" 2-morphisms:

1. Four 2-morphisms of essential type:

(a) Rank-two homotopy classes of bigons $\Sigma: \gamma_{1} \Rightarrow \gamma_{2}$ in $Y$ going between paths.

(b) Rank-one homotopy classes of paths $\Theta: \alpha \rightarrow \alpha^{\prime}$ in $Y^{[2]}$ considered as 2-isomorphisms

$$
\Theta: \alpha^{\prime} * \pi_{1}(\Theta) \Rightarrow \pi_{2}(\Theta) * \alpha,
$$

going between 1-morphisms mixed from jumps and paths. 
(c) Points $\Xi \in Y^{[3]}$ considered as 2-isomorphisms

$$
\Xi: \pi_{23}(\Xi) * \pi_{12}(\Xi) \Longrightarrow \pi_{13}(\Xi)
$$

going between jumps.

(d) Points $a \in Y$ considered as 2-isomorphisms

$$
\Delta_{a}: \mathrm{id}_{a}^{*} \Rightarrow(a, a)
$$

relating the formal identity with the trivial jump.

In (b) to (d) we demand that the 2-morphisms $\Theta, \Xi$ and $\Delta_{a}$ come with formal inverses, denoted by $\Theta^{-1}, \Xi^{-1}$ and $\Delta_{a}^{-1}$.

2. Two 2-morphisms of technical type:

(a) associators for the formal composition, i.e., 2-isomorphisms

$$
a_{\beta_{1}, \beta_{2}, \beta_{3}}^{*}:\left(\beta_{3} * \beta_{2}\right) * \beta_{1} \Rightarrow \beta_{3} *\left(\beta_{2} * \beta_{1}\right)
$$

for $\beta_{k}$ either paths or jumps, and unifiers

$$
l_{\beta}: \beta * \mathrm{id}_{a}^{*} \Rightarrow \beta \quad \text { and } \quad r_{\beta}: \mathrm{id}_{b}^{*} * \beta \Rightarrow \beta
$$

(b) for points $a \in Y$ and composable paths $\gamma_{1}$ and $\gamma_{2}$ 2-isomorphisms

$$
u_{a}^{*}: \operatorname{id}_{a} \Rightarrow \operatorname{id}_{a}^{*} \quad \text { and } \quad c_{\gamma_{1}, \gamma_{2}}^{*}: \gamma_{2} * \gamma_{1} \Rightarrow \gamma_{2} \circ \gamma_{1}
$$

expressing that the formal composition restricted to paths yields the usual composition of paths.

Now we consider the set which is freely generated from these basic 2-morphisms in virtue of a formal horizonal composition $*$ and a formal vertical composition $\circledast$. The formal identity 2-morphisms are denoted by $\mathrm{id}_{\beta}^{\circledast}: \beta \Rightarrow \beta$ for any 1 -morphism $\beta$. The set of 2-morphisms of the 2-category $\mathcal{P}_{2}^{\pi}(M)$ is this set subject to the following list of identifications:

(I) Identifications of 2-categorical type. The formal compositions $*$ and $\circledast$, and the 2-isomorphisms of type (2a) form the structure of a 2-category and we impose all identifications required by the axioms $(\mathrm{C} 1)$ to $(\mathrm{C} 4)$.

(II) Identifications of 2-functorial type. We have the structure of a 2-functor

$$
\iota: \mathcal{P}_{2}(Y) \rightarrow \mathcal{P}_{2}^{\pi}(M)
$$

This 2-functor regards points, paths and bigons in $Y$ as objects, 1-morphisms of type (1) and 2-morphisms of type (1a), respectively. Its compositors and unitors are the 2-isomorphisms $c^{*}$ and $u^{*}$ of type (2b). We impose all identification required by the axioms $(\mathrm{F} 1)$ to $(\mathrm{F} 4)$ for this 2 -functor. 
(III) Identifications of transformation type. We have the structure of a pseudonatural transformation

$$
\Gamma: \pi_{1}^{*} \iota \rightarrow \pi_{2}^{*} \iota
$$

between 2-functors defined over $Y^{[2]}$. Its component at a 1-morphism

$$
\Theta: \alpha \rightarrow \alpha^{\prime}
$$

in $\mathcal{P}_{1}\left(Y^{[2]}\right)$ is the 2-isomorphism $\Theta$ of type (1b). We impose all identifications required by the axioms (T1) and (T2) for this pseudonatural transformation.

(IV) Identification of modification type. We have the structure of a modification

$$
\pi_{23}^{*} \Gamma \circ \pi_{12}^{*} \Gamma \Rightarrow \pi_{13}^{*} \Gamma
$$

between pseudonatural transformations of 2-functors defined over $Y^{[3]}$. Its component at an object $\Xi \in Y^{[3]}$ is the 2-isomorphism $\Xi$ of type (1c). We have the structure of another modification

$$
\mathrm{id}_{\iota} \Rightarrow \Delta^{*} \Gamma
$$

between pseudonatural transformations of 2-functors over $Y$, whose component at an object $a \in Y$ is the 2-isomorphism $\Delta_{a}$ of type (1d). We impose all identifications required by the commutativity of diagram (A.4) for both modifications.

(V) Identifications of essential type:

1. For every point $\Psi \in Y^{[4]}$ we impose the commutativity of the diagram

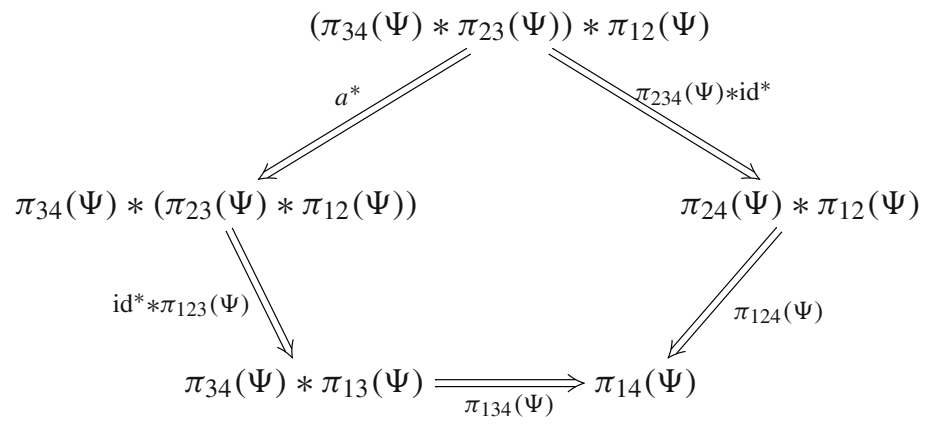

of compositions of jumps.

2. For every point $\alpha \in Y^{[2]}$ we impose the commutativity of the diagrams
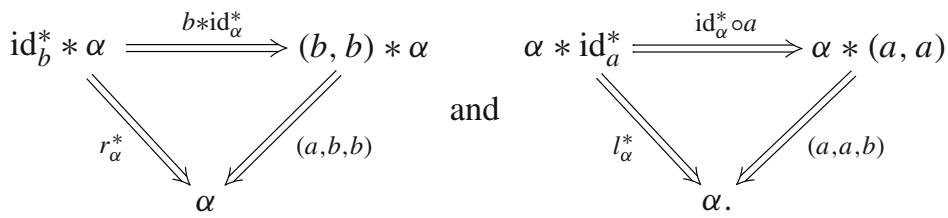
According to (I) we have defined a 2-category $\mathcal{P}_{2}^{\pi}(M)$.

Lemma 3.1.1 The 2-category $\mathcal{P}_{2}^{\pi}(M)$ is a 2-groupoid.

Proof All 2-morphisms except those of type (1a) are invertible by definition. But for a 2-morphism of type (1a), a bigon $\Sigma: \gamma \Rightarrow \gamma^{\prime}$, we have

$$
\Sigma^{-1} \circledast \Sigma \stackrel{(\mathrm{II})}{=} \Sigma^{-1} \bullet \Sigma=\mathrm{id}_{\gamma} \stackrel{(\mathrm{II})}{=} \mathrm{id}_{\gamma}^{\circledast},
$$

and analogously $\Sigma \circledast \Sigma^{-1}=\mathrm{id}_{\gamma^{\prime}}^{\circledast}$. Here we have used identification (II); more precisely axiom (F1) of the 2-functor $\iota: \mathcal{P}_{2}(Y) \rightarrow \mathcal{P}_{2}^{\pi}(M)$. To see that a path $\gamma: a \rightarrow b$ is invertible, we claim that $\gamma^{-1}$ is a weak inverse. It is easy to construct the 2-isomorphisms $i_{\gamma}$ and $j_{\gamma}$ using the 2-isomorphisms of type (2b). The required identities (A.1) for these 2-isomorphisms are then satisfied due to identification (II). To see that a jump $\alpha \in Y^{[2]}$ with $\alpha=(x, y)$ is invertible, we claim that $\bar{\alpha}:=(y, x)$ is a weak inverse. The 2-isomorphisms $i_{\alpha}$ and $j_{\alpha}$ can be constructed from 2-isomorphisms of types (1c) and (1d). The identities (A.1) are satisfied due to identifications (V1) and (V2).

We remark that we have a 2-functor $\iota: \mathcal{P}_{2}(Y) \hookrightarrow \mathcal{P}_{2}^{\pi}(M)$, a pseudonatural transformation $\Gamma$ and modifications (3.1.1) and (3.1.2) claimed by identifications (II), (III) and (IV).

\subsection{The Section 2-Functor}

There is a canonical strict 2-functor

$$
p^{\pi}: \mathcal{P}_{2}^{\pi}(M) \rightarrow \mathcal{P}_{2}(M)
$$

whose composition with the 2 -functor $\iota$ is equal to the 2 -functor $\pi_{*}: \mathcal{P}_{2}(Y) \rightarrow \mathcal{P}_{2}(M)$ induced from the projection, i.e.

$$
p^{\pi} \circ \iota=\pi_{*} .
$$

It sends all 1-morphisms and 2-morphisms which are not in the image of $\iota$ to identities. In this section we show the following result.

Proposition 3.2.1 The 2-functor $p^{\pi}$ is an equivalence of 2-categories.

In order to prove this we introduce an inverse 2 -functor

$$
s: \mathcal{P}_{2}(M) \rightarrow \mathcal{P}_{2}^{\pi}(M) .
$$

Since the 2-functor $p^{\pi}$ is surjective on objects, we call $s$ the section 2-functor. To define $s$, we lift points, paths and bigons in $M$ along the surjective submersion $\pi$, and use the jumps and the several 2-morphisms of the codescent 2-groupoid whenever no "global" lifts exist.

For preparation we need the following technical lemma. 
Lemma 3.2.2 Let $\gamma: x \rightarrow y$ be a path in $M$, and let $\tilde{x}, \tilde{y} \in Y$ be lifts of the endpoints, i.e. $\pi(\tilde{x})=x$ and $\pi(\tilde{y})=y$.

(a) There exists a 1-morphism $\tilde{\gamma}: \tilde{x} \rightarrow \tilde{y}$ in $\mathcal{P}_{2}^{\pi}(M)$ such that $p^{\pi}(\tilde{\gamma})=\gamma$.

(b) Let $\tilde{\gamma}: \tilde{x} \rightarrow \tilde{y}$ and $\tilde{\gamma}^{\prime}: \tilde{x} \rightarrow \tilde{y}$ be two such 1-morphisms. Then, there exists a unique 2-isomorphism $A: \tilde{\gamma} \Rightarrow \tilde{\gamma}^{\prime}$ in $\mathcal{P}_{2}^{\pi}(M)$ such that $p^{\pi}(A)=\mathrm{id}_{\gamma}$.

The assertion (a) is proven as [4, Lemma 2.15]. The proof of (b) requires some preparation.

Lemma 3.2.3 Let $p \in M$ be a point and $a, b \in Y$ with $\pi(a)=\pi(b)=p$. Let $\alpha: a \rightarrow b$ and $\beta: a \rightarrow b$ be 1-morphisms in $\mathcal{P}_{2}^{\pi}(M)$ which are compositions of jumps.

(a) There exists a 2-isomorphism $\Xi: \alpha \Rightarrow \beta$ with $p^{\pi}(\Xi)=\mathrm{id}_{\mathrm{id}_{p}}$.

(b) Any 2-isomorphism $\Xi: \alpha \Rightarrow \beta$ with $p^{\pi}(\Xi)=\operatorname{id}_{\mathrm{id}_{p}}$ can be represented by a composition of 2-morphisms of type (1c).

(c) The 2-isomorphism from (a) is unique.

Proof It is easy to construct the 2-isomorphism of (a) using only 2-isomorphisms of type (1c) and their inverses. To show (b) let $\Xi: \alpha \Rightarrow \beta$ be a 2 -isomorphism with $p^{\pi}(\Xi)=\mathrm{id}_{\mathrm{id}_{p}}$, represented by a composition of 2-morphisms of any type. In the following we draw pasting diagrams to demonstrate that all 2-morphisms of types (1a), (1b) and (1d) can subsequently be killed.

To prepare some machinery notice that identification (III) imposes axiom (T2) for the pseudonatural transformation $\Gamma$, which is, for any bigon $\Sigma: \Theta_{1} \Rightarrow \Theta_{2}$ in $Y^{[2]}$, the identity:

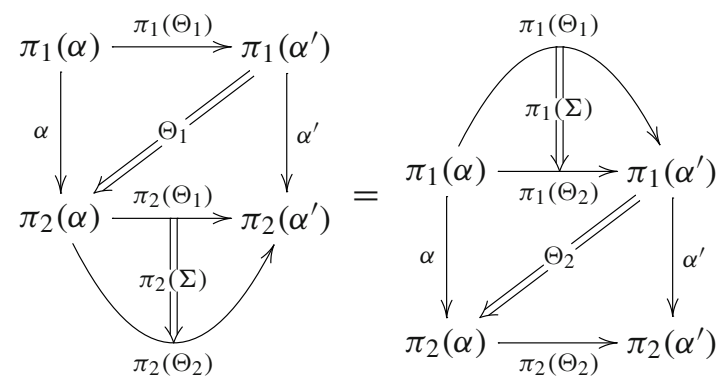

In the same way, identification (IV) imposes the axiom for the modification $\mathrm{id}_{\iota} \Rightarrow \Delta^{*} \Gamma$, which is, for any path $\gamma: a \rightarrow b$ in $Y$, the identity

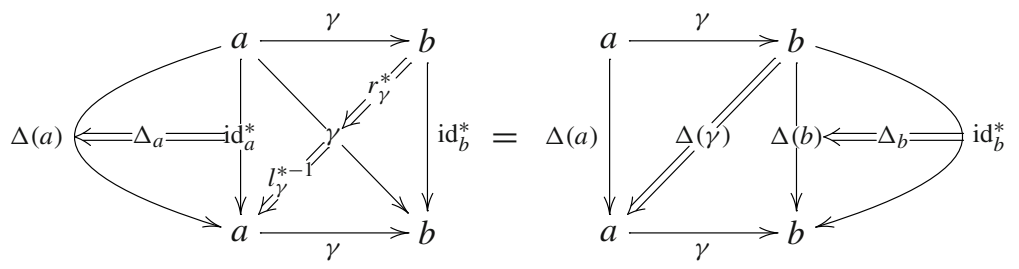


Using (3.2.3) we can write the identity 2-morphism associated to the path $\gamma$ in a very fancy way, namely

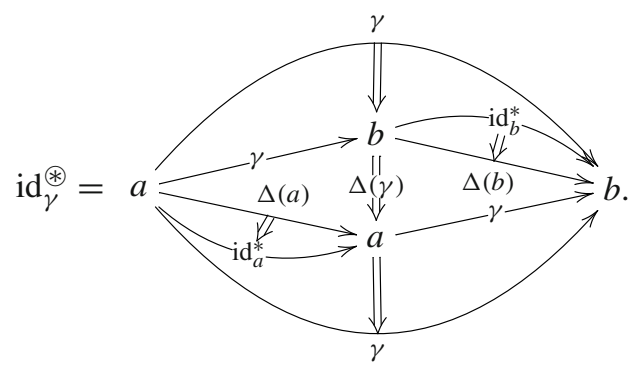

Now suppose that $\Sigma: \gamma \Rightarrow \gamma^{\prime}$ is some 2-morphism of type (1a) that we want to kill. We write $\Sigma=\Sigma \circledast \mathrm{id}_{\gamma}^{\circledast}$ and use (3.2.4). Using the naturality of the 2-morphism $l_{\gamma}^{*}$ claimed by identification (I) we have
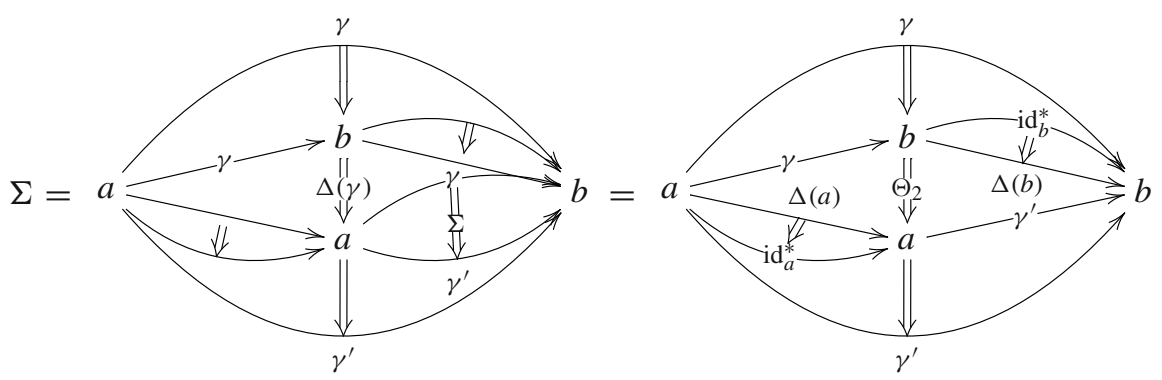

where the second identity is obtained from (3.2.2) by taking $\Theta_{1}:=\Delta(\gamma)$ and $\Theta_{2}:=\left(\gamma, \gamma^{\prime}\right)$ which is only possible because we have assumed that $p^{\pi}(\Sigma)=\mathrm{id}_{\mathrm{id}_{p}}$. We can thus kill every 2-morphism of type (1a).

Suppose now that $\Psi: \mu \Rightarrow v$ is a 2-morphism of type (1b). To kill it we need identification (IV), namely the axiom for the modification $\pi_{23}^{*} \Gamma \circ \pi_{12}^{*} \Gamma \Rightarrow \pi_{13}^{*} \Gamma$. For any path $\Theta: \Xi \rightarrow \Xi^{\prime}$ in $Y^{[3]}$, the corresponding pasting diagram is
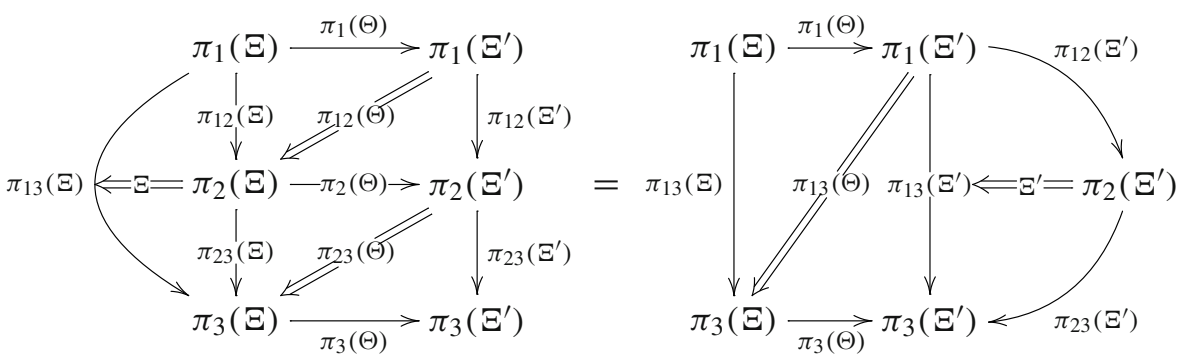

Here we suppress writing the associators and the bracketing of the 1-morphisms. Using this identity we have 


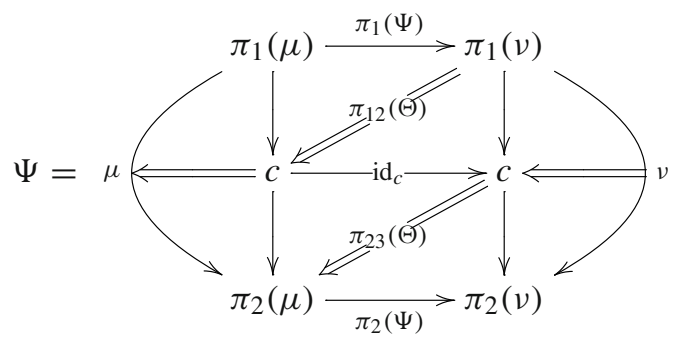

for $c \in Y$ an arbitrary point with $\pi(c)=p$ and $\Theta:=\left(\pi_{1}(\Psi), \operatorname{id}_{c}, \pi_{2}(\Psi)\right)$ which is only possible because $p^{\pi}(\Psi)=\mathrm{id}_{\mathrm{id}_{p}}$.

We can now assume that the 2-morphism $\Xi: \alpha \Rightarrow \beta$ we started with contains no 2-morphism of type (1a) and by (3.2.6) only those 2-morphism $\Theta=\left(\gamma_{1}, \gamma_{2}\right)$ for which either $\gamma_{1}$ or $\gamma_{2}$ is the identity path of the point $c$. If both $\gamma_{1}$ and $\gamma_{2}$ are identity paths, we can replace $\Theta$ according to (3.2.3) by two 2-morphisms of type (1d). It is now a combinatorial task to kill all 2-morphisms which start or end on paths, in particular all 2-morphisms of type (2b). Then one kills all 2-morphisms of types (1d) and the remaining unifiers $l_{\beta}^{*}$ and $r_{\beta}^{*}$. Finally, all associators $a^{*}$ can be killed using their naturality with respect to 2-morphisms of type (1c). This proves (b).

To prove (c) we assume that $\Xi^{\prime}: \alpha \Rightarrow \beta$ is any 2-isomorphism with $p^{\pi}(\Xi)=$ $\mathrm{id}_{\mathrm{id}_{p}}$. By (b) we can assume that $\Xi^{\prime}$ is composed only of 2-isomorphisms of type (1c). It is straightforward to see that two such compositions can be transformed into each other if six identities are satisfied: two bubble identities and four fusion identities. The two bubble identities are
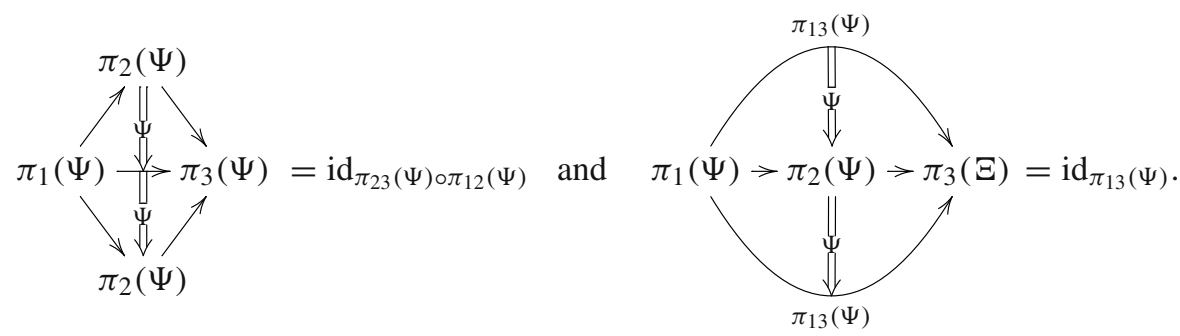

They follow from the fact that the 2-morphisms of type (1c) are invertible. The first fusion identity is identification (V1),

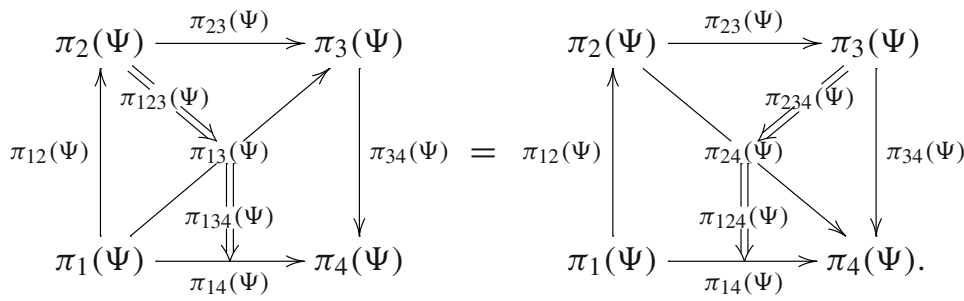


The other three fusion identities are analogous identities for formal inverses $\bar{\Psi}$ and mixtures of $\Psi$ and $\bar{\Psi}$.

Proof of Lemma 3.2.2 (b) Now let $\gamma: x \rightarrow y$ be a path in $M$, and let $\tilde{x}, \tilde{y} \in Y$ be lifts of the endpoints, i.e. $\pi(\tilde{x})=x$ and $\pi(\tilde{y})=y$. We compare the two lifts $\tilde{\gamma}$ and $\tilde{\gamma}^{\prime}$ of $\gamma$ in the following way. Let $P \subset M$ be the set of points over whose fibre either $\tilde{\gamma}_{1}$ or $\tilde{\gamma}_{2}$ has a jump. The set $P$ is finite and ordered by the orientation of the path $\gamma$, so that we may put $P=\left\{p_{0}, \ldots, p_{n}\right\}$ with $p_{0}=x$ and $p_{n}=y$. Now we write

$$
\gamma=\gamma_{n} \circ \cdots \circ \gamma_{1}
$$

for paths $\gamma_{k}: p_{k-1} \rightarrow p_{k}$. We also write $\tilde{\gamma}$ as a composition of lifts $\tilde{\gamma}_{k}: a_{k} \rightarrow b_{k}$ of $\gamma_{k}$ and (possibly multiple) jumps $b_{k} \rightarrow \alpha_{k+1}$ located over the points $p_{k}$; analogously for $\tilde{\gamma}^{\prime}$. This defines jumps $\alpha_{k}:=\left(a_{k}, a_{k}^{\prime}\right)$ and $\beta_{k}:=\left(b_{k}, b_{k}^{\prime}\right)$. Now, over the paths $\gamma_{k}$, we take 2-isomorphisms

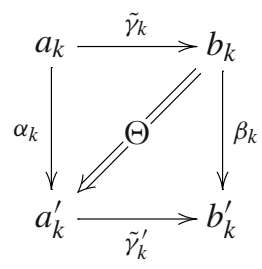

with $\Theta:=\left(\tilde{\gamma}_{k}, \tilde{\gamma}_{k}^{\prime}\right)$. Over the points $p_{k}$ we need 2-isomorphisms of the form
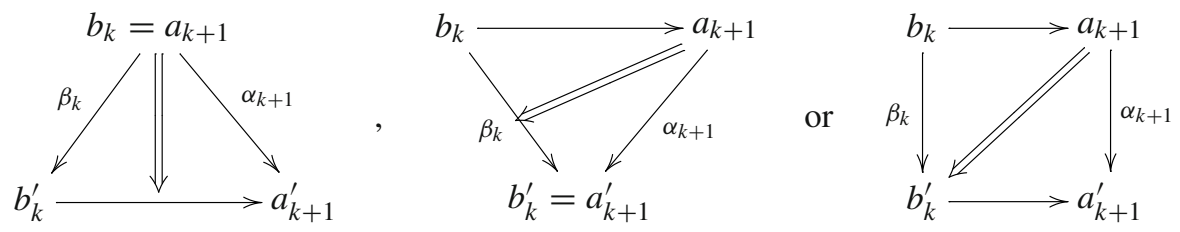

the first whenever $\tilde{\gamma}^{\prime}$ has jumps over $p_{k}$ and $\tilde{\gamma}$ has not, the second whenever $\tilde{\gamma}$ has jumps and $\tilde{\gamma}^{\prime}$ has not, and the third whenever both lifts have jumps. By Lemma 3.2.3 these 2-isomorphisms exist and are unique. Then, all of the four diagrams above can be put next to each other; this defines a 2-isomorphism $\tilde{\gamma} \Rightarrow \tilde{\gamma}^{\prime}$. We call the 2-morphism constructed like this the canonical 2-morphism.

It remains to show that every 2-morphism $A: \tilde{\gamma} \Rightarrow \tilde{\gamma}^{\prime}$ with $p^{\pi}(A)=\mathrm{id}_{\gamma}$ is equal to this canonical 2-morphism. First, we kill all bigons contained in $A$ by the argument given in the proof of Lemma 3.2.3. We consider two cases:

1. A contains no paths except those contained in $\tilde{\gamma}$ or $\tilde{\gamma}^{\prime}$. In this case $A$ is already equal to the canonical 2-morphism. Namely, each of the pieces $\tilde{\gamma}_{k}$ or $\tilde{\gamma}_{k}^{\prime}$ can only be target or source of a 2-morphism of type (1b). These are now necessarily the pieces (3.2.7). It remains to consider the 2-morphisms between the jumps. But these are by Lemma 3.2.3 equal to the pieces (3.2.8). This shows that $A$ is the canonical 2-morphism. 
2. There exists a path $\gamma_{0}$ in $\mathcal{P}_{2}^{\pi}(M)$ which is target or source of some 2-morphism contained in $A$ but not contained in $\tilde{\gamma}$ or $\tilde{\gamma}^{\prime}$. In this case there exists a 1-morphism $\tilde{\gamma}_{o}: \tilde{x} \rightarrow \tilde{y}$ together with 2-morphisms $A_{1}: \tilde{\gamma} \Rightarrow \tilde{\gamma}_{0}$ and $A_{2}: \tilde{\gamma}_{0} \Rightarrow \tilde{\gamma}^{\prime}$ such that $A=A_{2} \bullet A_{1}$. By iteration, we can decompose $A$ in a vertical composition of 2-morphisms which fall into case 1 , i.e. into a vertical composition of canonical 2-morphisms.

It remains to conclude with the observation that the vertical composition $A_{2} \bullet A_{1}$ of two canonical 2-morphisms is again canonical.

To construct the 2-functor $s$ we fix an open cover $\left\{U_{i}\right\}_{i \in I}$ of $M$ together with smooth sections $\sigma_{i}: U_{i} \rightarrow Y$, we fix choices of lifts $s(p) \in Y$ for all points $p \in M$, and we fix for every path $\gamma: x \rightarrow y$ in $M$ a 1-morphism $s(\gamma): s(x) \rightarrow s(y)$ in $\mathcal{P}_{2}^{\pi}(M)$. Such lifts exist according to Lemma 3.2.2 (a). For the identity 1-morphisms $\operatorname{id}_{x}$ we choose the identity 1-morphisms $\operatorname{id}_{s(x)}^{*}$. Moreover, we require $s\left(\gamma^{-1}\right)=s(\gamma)^{-1}$, meaning that $s(\gamma)^{-1}$ is the reverse order composition of the inverses $\tilde{\gamma}^{-1}$ of the involved paths $\tilde{\gamma}$, and of the inverses $\bar{\alpha}$ of all involved jumps $\alpha$. These choices define $s$ on objects and 1-morphisms.

Now let $\Sigma: \gamma_{1} \Rightarrow \gamma_{2}$ be a bigon in $M$. Its image $\Sigma\left([0,1]^{2}\right) \subset M$ is compact and hence covered by open sets indexed by a finite subset $J \subset I$. We choose a decomposition of $\Sigma$ in a vertical and horizontal composition of bigons $\left\{\Sigma_{j}\right\}_{j \in J}$ such that $\Sigma_{j}\left([0,1]^{2}\right) \subset U_{j}$. Then we define $s(\Sigma)$ to be composed from 2-morphisms $s\left(\Sigma_{j}\right)$ in the same way as $\Sigma$ was composed from the $\Sigma_{j}$. It thus remains to define the 2-functor $s$ on bigons $\Sigma$ which are contained in an open set $U$ which has a section $\sigma: U \rightarrow Y$. We define for such a bigon
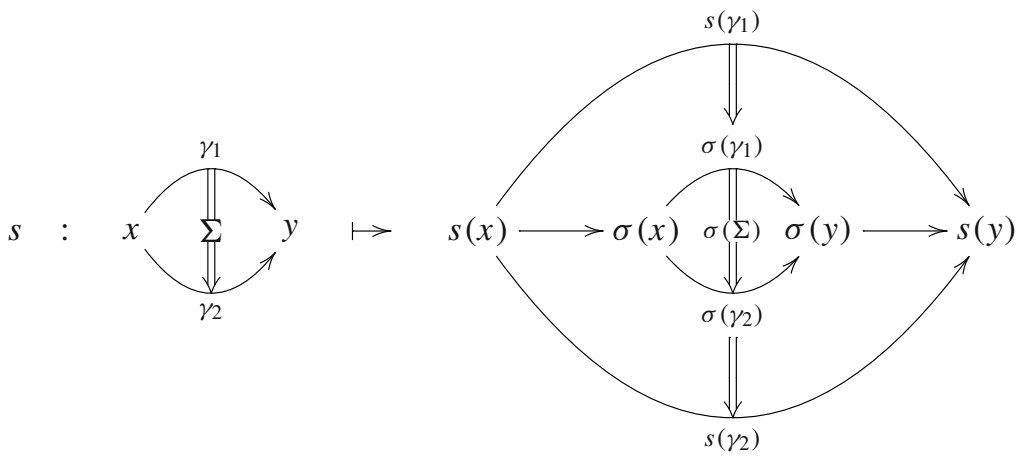

where the unlabelled 1-morphisms are the obvious jumps, and the unlabelled 2morphisms are the unique 2-isomorphisms from Lemma 3.2.2 (b).

The 2-functor $s: \mathcal{P}_{2}(M) \rightarrow \mathcal{P}_{2}^{\pi}(M)$ whose structure we have defined above is not strict. While its unitor is trivial because we have by definition $s\left(\mathrm{id}_{x}\right)=\mathrm{id}_{s(x)}^{*}$, its compositor $c_{\gamma_{1}, \gamma_{2}}: s\left(\gamma_{2}\right) \circ s\left(\gamma_{1}\right) \Rightarrow s\left(\gamma_{2} \circ \gamma_{1}\right)$ is defined to be the unique 2isomorphism from Lemma 3.2.2 (b). All axioms for the 2-functor $s$ follow from the uniqueness of these 2-isomorphisms.

For later purpose, we note the following consequence of the definitions. 
Lemma 3.2.4 If $\gamma: x \rightarrow y$ is a path in $M$, then the compositor $c_{\gamma, \gamma^{-1}}$ of $s$ is a composition of 2-morphisms of types (2a) and (2b), type (1d), and those 2-morphisms $\Xi \in Y^{[3]}$ of type (1c) that are in the image of $\Delta_{121}: Y^{[2]} \rightarrow Y^{[3]}:(a, b) \mapsto(a, b, a)$.

Now we can proceed with the remaining proof of the main result of this section.

Proof of Proposition 3.2.1 By construction we have $p^{\pi} \circ s=\mathrm{id}_{\mathcal{P}_{2}(M)}$. It remains to construct a pseudonatural equivalence

$$
\zeta: s \circ p^{\pi} \rightarrow \operatorname{id}_{\mathcal{P}_{2}^{\pi}(M)}
$$

We define $\zeta$ on both basic 1-morphisms. Its component at a path is

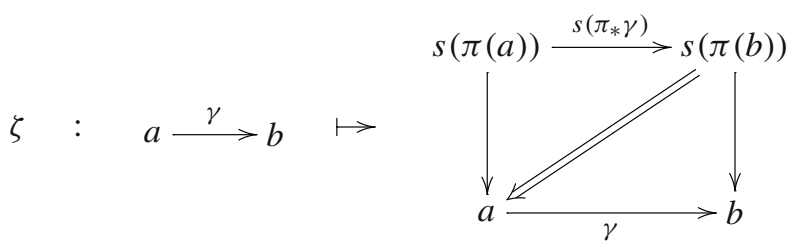

where the unlabelled 1-morphisms are again the obvious jumps, and the 2-isomorphism is the unique one. If $s\left(\pi_{*} \gamma\right)$ happens to be just a path, this 2-isomorphism is just of type (1b). The component of $\zeta$ at a jump is

$$
\zeta \quad: \quad \pi_{1}(\alpha) \stackrel{\alpha}{\longrightarrow} \pi_{2}(\alpha) \quad \mapsto
$$

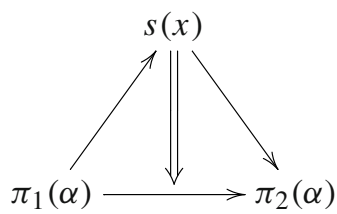

with $x:=\pi\left(\pi_{1}(\alpha)\right)=\pi\left(\pi_{2}(\alpha)\right)$; this is just one 2-isomorphism of type (1c). For some general 1-morphism, $\zeta$ puts the 2-isomorphisms above next to each other; this way axiom (T1) is automatically satisfied. Axiom (T2) follows again from the uniqueness of the 2-morphisms we have used.

In order to show that $\zeta$ is invertible we need to find another pseudonatural transformation $\xi: \operatorname{id}_{\mathcal{P}_{2}^{\pi}(M)} \rightarrow s \circ p^{\pi}$ together with invertible modifications $i_{\zeta}: \xi \circ \zeta \Longrightarrow \mathrm{id}_{s \circ p^{\pi}}$ and $j_{\zeta}: \operatorname{id}_{\mathrm{id}_{\mathcal{P}_{2}^{\pi}(M)}} \Rightarrow \zeta \circ \xi$ that satisfy the zigzag identities. The pseudonatural transformation $\xi$ can be defined in the same way as $\zeta$ just by turning the diagrams upside down, using the formal inverses. The modifications $i_{\zeta}$ and $j_{\zeta}$ assign to a point $a \in Y$ the 2-isomorphisms 

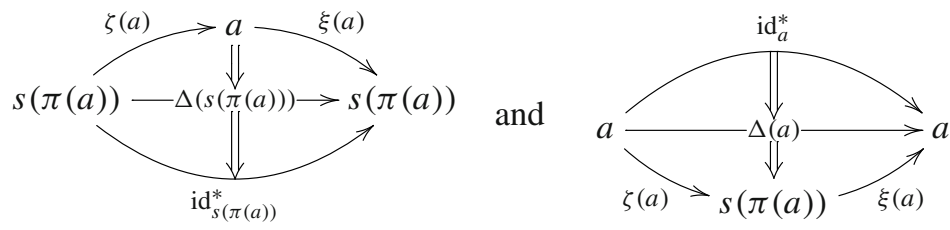

that combine 2-isomorphisms of type (1c) and (1d). The zigzag identities are satisfied due to the uniqueness of 2-isomorphisms we have used.

Corollary 3.2.5 The section 2-functor $s: \mathcal{P}_{2}(M) \rightarrow \mathcal{P}_{2}^{\pi}(M)$ is independent (up to pseudonatural equivalence) of all choices, namely the choice of lifts of points and 1-morphisms, the choice of the open cover, and the choice of local sections.

This follows from the fact that two weak inverses of a 1-morphism in a 2-category are automatically 2-isomorphic.

\subsection{Pairing with descent data}

In this section we relate the codescent 2-groupoid $\mathcal{P}_{2}^{\pi}(M)$ to the descent 2-category $\mathfrak{D e s}_{\pi}^{2}(i)$ defined in Sect. 2.2 in terms of a strict 2-functor

$$
R: \mathfrak{D e s}_{\pi}^{2}(i) \rightarrow \operatorname{Funct}\left(\mathcal{P}_{2}^{\pi}(M), T\right)
$$

This 2-functor expresses that the 2-groupoid $\mathcal{P}_{2}^{\pi}(M)$ is " $T$-dual" to the descent 2category; this justifies the term codescent 2-groupoid.

The 2-functor $R$ labels the structure of the codescent 2-groupoid by descent data in a natural way. To start with, let (triv, $g, \psi, f$ ) be a descent object. Its image under $R$ is a 2 -functor

$$
R_{(\text {triv }, g, \psi, f)}: \mathcal{P}_{2}^{\pi}(M) \rightarrow T
$$

defined as follows. To an object $a \in Y$ it assigns the object $\operatorname{triv}_{i}(a)$ in $T$. On basic 1-morphisms it is defined by the following assignments:

$$
\begin{aligned}
& a \stackrel{\gamma}{\longrightarrow} a^{\prime} \mapsto \quad \operatorname{triv}_{i}(a) \stackrel{\operatorname{triv}_{i}(\gamma)}{\longrightarrow} \operatorname{triv}_{i}\left(a^{\prime}\right) \\
& \pi_{1}(\alpha) \stackrel{\alpha}{\longrightarrow} \pi_{2}(\alpha) \quad \mapsto \quad \pi_{1}^{*} \operatorname{triv}_{i}(\alpha) \stackrel{g(\alpha)}{\longrightarrow} \pi_{2}^{*} \operatorname{triv}_{i}(\alpha) .
\end{aligned}
$$

To a formal composition of basic 1-morphisms it assigns the composition of the respec-

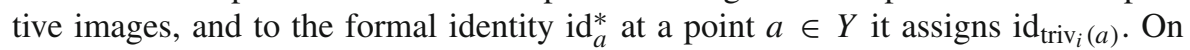


the basic 2-morphisms of essential types (1a) to (1d) it is defined by the following assignments:
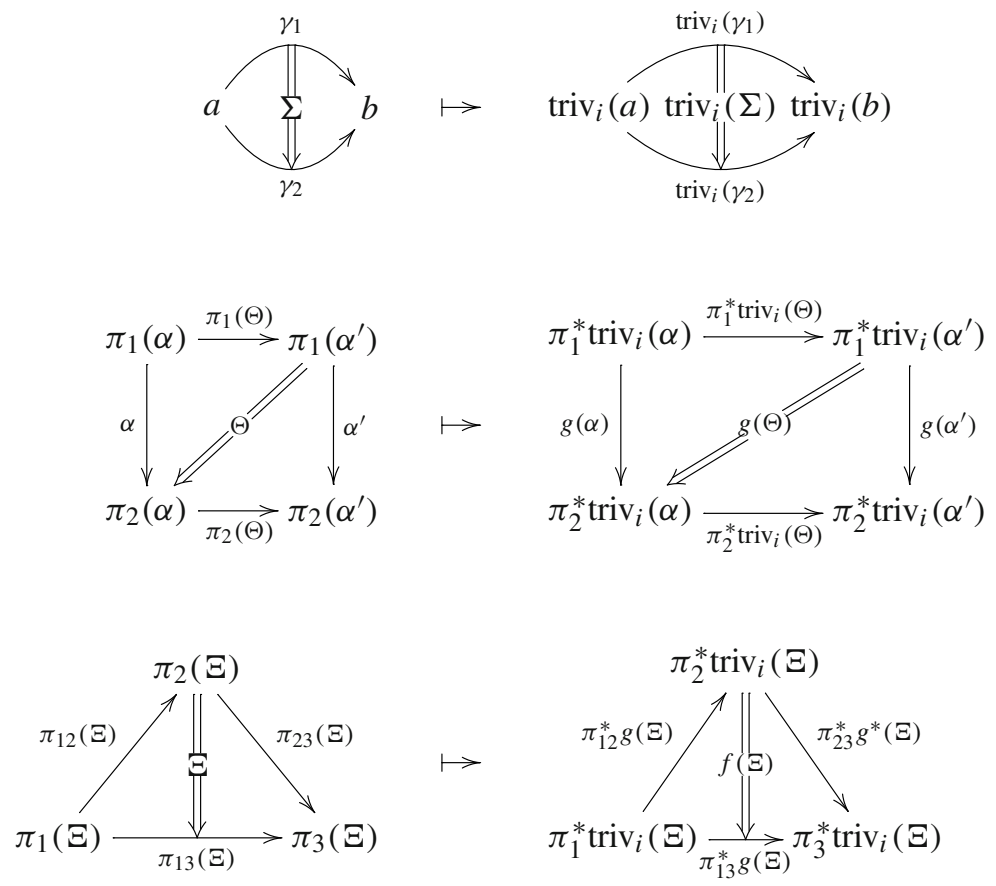

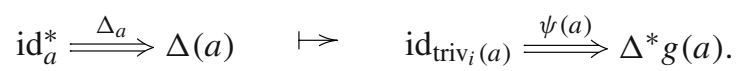

To the basic 2-morphisms of technical type (2a) it assigns associators and unifiers of the 2-category $T$. To those of type (2b) it assigns unitors and compositors of the 2 -functor $i$, i.e.

$$
\begin{aligned}
& \operatorname{id}_{a} \stackrel{u_{a}^{*}}{\Longrightarrow} \operatorname{id}_{a}^{*} \mapsto \operatorname{triv}_{i}\left(\operatorname{id}_{a}\right) \stackrel{u_{\text {triv }(a)}^{i}}{\Longrightarrow} \operatorname{id}_{\operatorname{triv}_{i}(a)} \\
& \gamma_{2} * \gamma_{1} \stackrel{c_{\gamma_{1}, \gamma_{2}}^{*}}{\Longrightarrow} \gamma_{2} \circ \gamma_{1} \mapsto \operatorname{triv}_{i}\left(\gamma_{2}\right) \circ \operatorname{triv}_{i}\left(\gamma_{1}\right) \stackrel{c_{\operatorname{triv}\left(\gamma_{1}\right), \operatorname{triv}\left(\gamma_{2}\right)}^{i}}{\Longrightarrow} \operatorname{triv}_{i}\left(\gamma_{2} \circ \gamma_{1}\right) .
\end{aligned}
$$

Finally, some formal horizontal and vertical composition of 2-morphisms is assigned to the composition of the images of the respective basic 2-morphisms, the formal horizontal composition replaced by the horizontal composition $\circ$ of $T$, and the formal vertical composition replaced by the vertical composition $\bullet$ of $T$.

By construction, all these assignments are well-defined under the identifications we have declared under the 2-morphisms of $\mathcal{P}_{2}^{\pi}(M)$ : 
- They are well-defined under the identifications (I) due to the axioms of the 2category $T$.

- They are well-defined under identifications (II) due to the axioms of the 2-functors triv and $i$.

- They are well-defined under identifications (III) due to the axioms of the pseudonatural transformation $g$.

- They are well-defined under identifications (IV) due to the axioms of the modifications $\psi$ and $f$.

- They are well-defined under the identifications (V) because these are explicitly assumed in the definition of descent objects, see diagrams (2.2.1) and (2.2.2).

We have now defined the 2-functor $R_{(\text {triv }, g, \psi, f)}$ on descent objects, 1-morphisms and 2-morphisms. Since for all points $a \in Y$

$$
R_{(\operatorname{triv}, g, \psi, f)}\left(\operatorname{id}_{a}^{*}\right)=\operatorname{id}_{\operatorname{triv}_{i}(a)}=\operatorname{id}_{R_{(\operatorname{triv}, g, \psi, f)}(a)},
$$

it has a trivial unitor. Furthermore,

$$
R_{(\operatorname{triv}, g, \psi, f)}(\gamma) \circ R_{(\operatorname{triv}, g, \psi, f)}(\beta)=R_{(\operatorname{triv}, g, \psi, f)}(\gamma * \beta)
$$

for all composable 1-morphisms $\beta$ and $\gamma$ of any type, so that it also has a trivial compositor. Hence, the 2-functor $R_{(\operatorname{triv}, g, \psi, f)}$ is strict, and it is straightforward to see that the remaining axioms (F1) and $(\mathrm{F} 2)$ are satisfied.

So far we have introduced a 2-functor associated to each descent object. Let us now introduce a pseudonatural transformation

$$
R_{(h, \varepsilon)}: R_{(\operatorname{triv}, g, \psi, f)} \rightarrow R_{\left(\operatorname{triv}^{\prime}, g^{\prime}, \psi^{\prime}, f^{\prime}\right)}
$$

associated to a descent 1-morphism

$$
(h, \varepsilon):(\operatorname{triv}, g, \psi, f) \rightarrow\left(\operatorname{triv}^{\prime}, g^{\prime}, \psi^{\prime}, f^{\prime}\right)
$$

Its definition is as natural as the one of the 2-functor given before. Its component at an object $a \in Y$ is the 1-morphism

$$
h(a): \operatorname{triv}_{i}(a) \rightarrow \operatorname{triv}_{i}^{\prime}(a) .
$$

Its components at basic 1-morphisms are given by the following assignments:

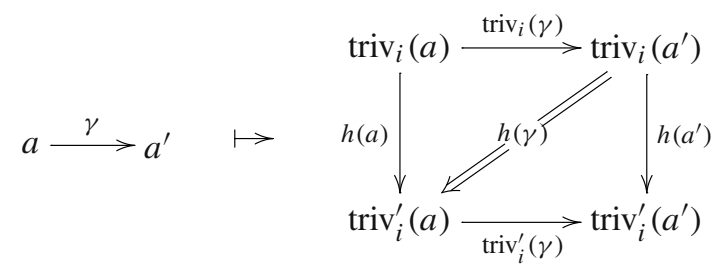




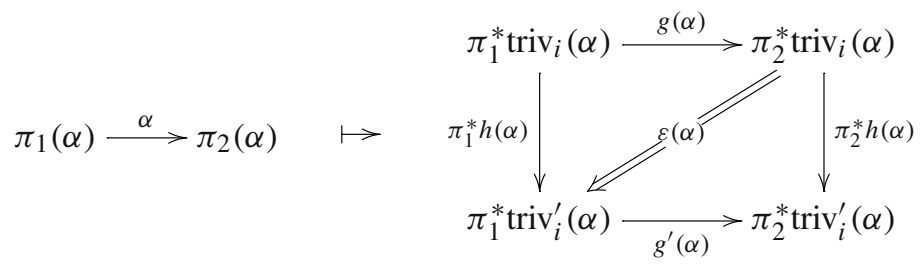

For compositions of 1-morphisms, $R_{(h, \varepsilon)}$ puts the diagrams for the involved basic 1morphisms next to each other. For example, to a composition $\gamma * \alpha$ of a jump $\alpha=(x, y)$ with a path $\gamma: y \rightarrow z$ it assigns the 2-isomorphism

$$
h(z) \circ\left(\operatorname{triv}_{i}(\gamma) \circ g(\alpha)\right) \Rightarrow\left(\operatorname{triv}_{i}^{\prime}(\gamma) \circ g(\alpha)\right) \circ h(x)
$$

which is (up to the obvious associators) obtained by first applying $h(\gamma)$ and then $\varepsilon(\alpha)$. This way, axiom (T1) for the pseudonatural transformation $R_{(h, \varepsilon)}$, namely the compatibility with the composition of 1-morphisms, is automatically satisfied. It remains to prove the following.

Lemma 3.3.1 The assignments $R_{(h, \varepsilon)}$ are compatible with the 2-morphisms of the codescent 2-groupoid in the sense of axiom (T2).

Proof We check the compatibility separately for each basic 2-morphism. For the essential 2-morphisms it comes from the following properties of the descent 1-morphism $(h, \varepsilon)$ :

- For type (1a) it comes from axiom (T2) for the pseudonatural transformation $h$.

- For type (1b) it comes from the axiom for the modification $\varepsilon$ and from axiom (T2) for the pseudonatural transformation $h$.

- For types (1c) and (1d) it comes from the conditions (2.2.3) and (2.2.4) on the descent 1-morphism $(h, \varepsilon)$.

For the technical 2-morphisms it comes from properties of the 2-category $T$ and the one of the 2-functor $i$ : for type (2a) it is satisfied because the associators and unifiers of $T$ are natural, and for type (2b) it is satisfied because the compositors and unitors of $i$ are natural.

We have now described a 2-functor associated to each descent object and a pseudonatural transformation associated to each descent 1-morphism. Now let (triv, $g, \psi, f$ ) and (triv', $\left.g^{\prime}, \psi^{\prime}, f^{\prime}\right)$ be descent objects and let $\left(h_{1}, \varepsilon_{1}\right)$ and $\left(h_{2}, \varepsilon_{2}\right)$ be two descent 1-morphisms between these. For a descent 2-morphism $E:\left(h_{1}, \varepsilon_{1}\right) \Longrightarrow\left(h_{2}, \varepsilon_{2}\right)$ we introduce now a modification

$$
R_{E}: R_{\left(h_{1}, \varepsilon_{1}\right)} \Rightarrow R_{\left(h_{2}, \varepsilon_{2}\right)} \text {. }
$$

Its component at an object $a \in Y$ is the 2-morphism $E(a): h_{1}(a) \Rightarrow h_{2}(a)$. The axiom for $R_{E}$, the compatibility with 1-morphisms, is satisfied for paths because $E$ is a modification, and for jumps because of the diagram (2.2.5) in the definition of descent 2-morphisms.

It is now straightforward to see the following statement. 
Proposition 3.3.2 The assignments defined above furnish a strict 2-functor

$$
R: \mathfrak{D e s}_{\pi}^{2}(i) \rightarrow \operatorname{Funct}\left(\mathcal{P}_{2}^{\pi}(M), T\right)
$$

The 2-functor $R$ "represents" the descent 2-category in a 2-category of 2functors; in fact in a faithful way. We recall from Sect. 2.2 that there is a 2-functor $V: \mathfrak{D e s}_{\pi}^{2}(i) \rightarrow \operatorname{Funct}\left(\mathcal{P}_{2}(Y), T\right)$ which is also a representation of the same kind (but not faithful). The relation between these two representations is the following observation.

Lemma 3.3.3 $\iota^{*} \circ R=V$, where $\iota^{*}$ denotes the composition with

$$
\iota: \mathcal{P}_{2}(Y) \rightarrow \mathcal{P}_{2}^{\pi}(M) \text {. }
$$

From this point of view, the codescent 2-groupoid enlarges the path 2-groupoid $\mathcal{P}_{2}(Y)$ by additional 1-morphisms (the jumps) and additional 2-morphisms in such a way that it carries a faithful representation of the descent 2-category.

Now we put the two main aspects of the codescent 2-groupoid together, namely the representation 2-functor $R$, and its equivalence with the path 2-groupoid in terms of the section 2-functor $s$ : the reconstruction 2-functor

$$
\operatorname{Rec}_{\pi}: \mathfrak{D e s}_{\pi}^{2}(i) \rightarrow \operatorname{Triv}_{\pi}^{2}(i)
$$

is defined to be the composition

$$
\mathfrak{D e s}_{\pi}^{2}(i) \stackrel{R}{\longrightarrow} \operatorname{Funct}\left(\mathcal{P}_{2}^{\pi}(M), T\right) \stackrel{s^{*}}{\longrightarrow} \operatorname{Funct}\left(\mathcal{P}_{2}(M), T\right)
$$

Here, $s^{*}$ is the composition with $s$. According to Corollary 3.2.5, the reconstruction 2 -functor is (up to pseudonatural equivalence) canonically attached to the surjective submersion $\pi: Y \rightarrow M$ and the 2-functor $i: \mathrm{Gr} \rightarrow T$.

In order to show that the reconstruction ends in the sub-2-category $\operatorname{Triv}_{\pi}^{2}(i)$ of $\operatorname{Funct}\left(\mathcal{P}_{2}(M), T\right)$ it remains to equip, for each descent object (triv, $\left.g, \psi, f\right)$, the reconstructed 2-functor

$$
F:=R_{(\operatorname{triv}, g, \psi, f)} \circ s
$$

with a $\pi$-local $i$-trivialization (triv, $t$ ). Clearly, we take the given 2 -functor triv as the first ingredient and are left with the construction of a pseudonatural equivalence

$$
t: \pi^{*} F \rightarrow \operatorname{triv}_{i}
$$

This equivalence is simply defined by 


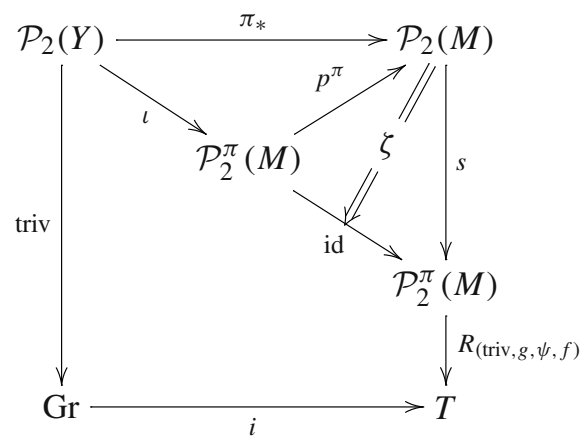

where $\zeta$ is the pseudonatural equivalence from Sect. 3.2. The triangle on the top of the latter diagram is Eq. (3.2.1), and the remaining subdiagram expresses the equation

$$
\iota^{*} R_{(\operatorname{triv}, g, \psi, f)}=\operatorname{triv}_{i}
$$

which follows from Lemma 3.3.3.

We conclude with a lemma about the reconstruction of 2-functors from normalized descent objects.

Lemma 3.3.4 Suppose $T$ is a strict 2-category, $i: \mathrm{Gr} \rightarrow T$ is a strict 2-functor, and (triv, $g, \psi, f$ ) is a normalized descent object. Then, the reconstructed 2-functor

$$
R_{\text {(triv, } g, \psi, f)} \circ S: \mathcal{P}_{2}(M) \rightarrow T
$$

is normalized in the sense explained in "Appendix 1".

Proof We write $R:=R_{(\operatorname{triv}, g, \psi, f)}$ and $F:=R \circ s$ for abbreviation. Let $x \in M$. Since $R$ is strict and $s$ has trivial unitor, $F$ has a trivial unitor:

$$
u_{x}^{F}=u_{s(x)}^{R} \bullet R\left(u_{x}^{s}\right)=R\left(\operatorname{id}_{\mathrm{id}_{s(x)}^{*}}\right)=\mathrm{id}_{R\left(\mathrm{id}_{s(x)}^{*}\right)}=\mathrm{id}_{\mathrm{id}_{\operatorname{triv}_{i}(s(x))}} .
$$

Let $\gamma: x \rightarrow y$ be a path. Since $R$ is strict, we have

$$
c_{\gamma, \gamma^{-1}}^{F}=R\left(c_{\gamma, \gamma^{-1}}^{s}\right)
$$

By Lemma 3.2.4 the compositor $c_{\gamma, \gamma^{-1}}^{s}$ consists of 2-morphisms of types (2a), (2b), (1c) and (1d). Since $T$ and $i$ are strict, all 2-morphisms of technical type (2a) and (2b) are sent by $R$ to identities. The 2-morphisms of types (1c) and (1d) are sent by $R$ to the components of $\psi$ and $f$, and these are identities since (triv, $g, \psi, f$ ) is a normalized descent object. 


\section{Local: global equivalence}

In this section we prove the main theorem of this article, namely that extraction and reconstruction establish an equivalence between locally defined descent data and globally defined 2-functors.

\subsection{Equivalence for a fixed cover}

Let $i: \mathrm{Gr} \rightarrow T$ be a 2-functor from a strict 2-groupoid $\mathrm{Gr}$ to a 2-category $T$, and let $\pi: Y \rightarrow M$ be a surjective submersion.

Proposition 4.1.1 The 2-functor

$$
\operatorname{Ex}_{\pi}: \operatorname{Triv}_{\pi}^{2}(i) \rightarrow \mathfrak{D e s}_{\pi}^{2}(i)
$$

is an equivalence of 2-categories.

For the proof we shall choose a Sect. 2-functor $s: \mathcal{P}_{2}(M) \rightarrow \mathcal{P}_{2}^{\pi}(M)$ defining the reconstruction 2-functor $\operatorname{Rec}_{\pi}$. We show that $\mathrm{Ex}_{\pi}$ and $\operatorname{Rec}_{\pi}$ form an equivalence of 2-categories. This is done in the following two lemmata.

Lemma 4.1.2 There is a pseudonatural equivalence $\operatorname{Ex}_{\pi} \circ \operatorname{Rec}_{\pi} \cong \operatorname{id}_{\mathfrak{D e s}_{\pi}^{2}(i)}$.

Proof Given a descent object (triv, $g, \psi, f$ ) let us pass to the reconstructed 2-functor and extract its descent data $\left(\operatorname{triv}^{\prime}, g^{\prime}, \psi^{\prime}, f^{\prime}\right)$. We find immediately triv' $=$ triv. Furthermore, the pseudonatural transformation $g^{\prime}$ has the components

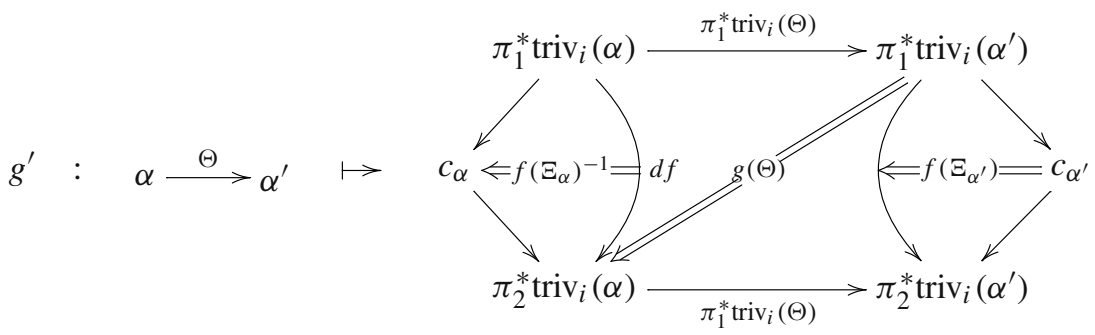

where we have introduced an object $c_{\alpha}:=\operatorname{triv}_{i}(s(p))$ where $p=\pi\left(\pi_{1}(\alpha)\right)=$ $\pi\left(\pi_{2}(\alpha)\right)$ and a 2-morphism $\Xi_{\alpha}:=\left(\pi_{1}(\alpha), s(p), \pi_{2}(\alpha)\right)$. It is useful to notice that this means that $f$ is a modification $f: g^{\prime} \Rightarrow g$. The modification $\psi^{\prime}$ has the component

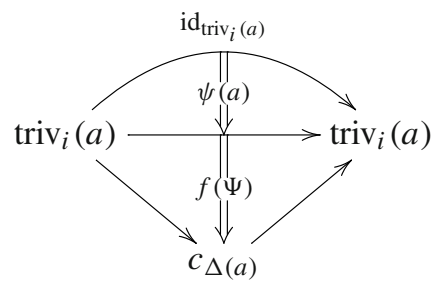


at a point $a \in Y$. Finally, the modification $f^{\prime}$ has the component

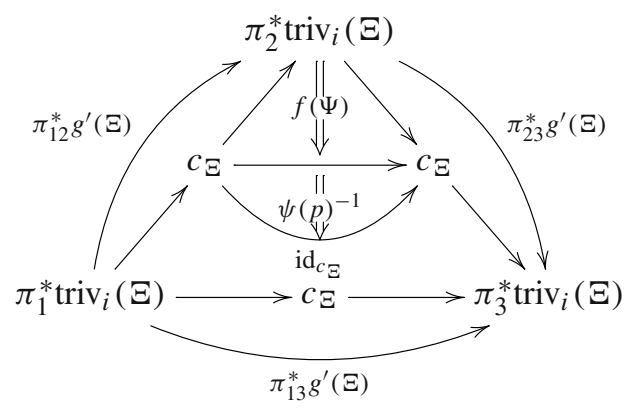

at a point $\Xi \in Y^{[3]}$, where we have introduced the 2-morphism $\Psi:=\left(c_{\Xi}, \pi_{2}^{*} \operatorname{triv}_{i}(\Xi)\right.$, $c_{\Xi}$ ), and $p$ is again the projection of $\Xi$ to $M$. Now it is straightforward to construct a descent 1-morphism

$$
\rho_{(\text {triv }, g, \psi, f)}:\left(\text { triv }, g^{\prime}, \psi^{\prime}, f^{\prime}\right) \rightarrow(\text { triv }, g, \psi, f)
$$

which consists of the identity pseudonatural transformation $h:=\mathrm{id}_{\text {triv }}$ and of a modification $\varepsilon: \pi_{2}^{*} h \circ g^{\prime} \Rightarrow g \circ \pi_{1}^{*} h$ induced from the modification $f: g^{\prime} \Rightarrow g$ and the left and right unifiers. This descent 1-morphism is the component of the pseudonatural equivalence $\rho: \operatorname{Ex}_{\pi} \circ \operatorname{Rec}_{\pi} \rightarrow$ id we have to construct, at the object (triv, $g, \psi, f$ ).

Let us now define the component of $\rho$ at a descent 1-morphism

$$
(h, \varepsilon):\left(\operatorname{triv}_{1}, g_{1}, \psi_{1}, f_{1}\right) \rightarrow\left(\operatorname{triv}_{2}, g_{2}, \psi_{2}, f_{2}\right)
$$

It is useful to introduce a modification $\tilde{\epsilon}: \bar{g}_{2} \circ \pi_{2}^{*} h \circ g_{1} \Rightarrow \pi_{1}^{*} h$ where $\bar{g}_{2}$ is the pullback of $g_{2}$ along the map $Y^{[2]} \rightarrow Y^{[2]}$ that exchanges the components. It is defined as the following composition of modifications:

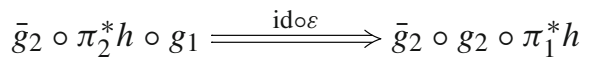

$$
\begin{aligned}
& \Downarrow \Delta_{121}^{*} f_{2} \text { oid }
\end{aligned}
$$

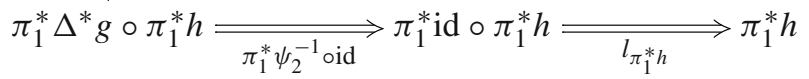

Now, if we reconstruct and extract local data $\left(h^{\prime}, \varepsilon^{\prime}\right)$, the pseudonatural transformation $h^{\prime}$ has the components 


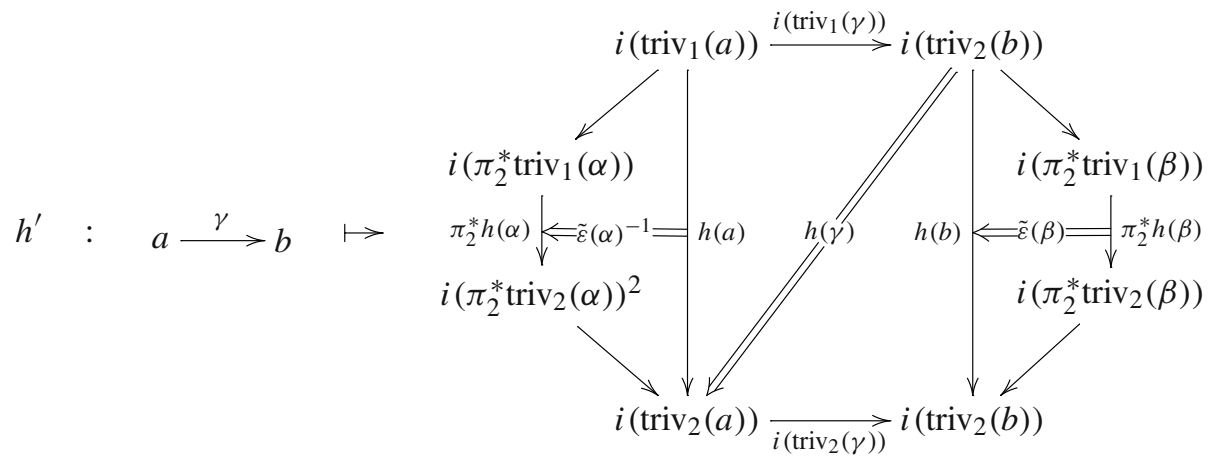

with $\alpha:=(a, s(\pi(a)))$ and $\beta=(b, s(\pi(b)))$. Like above we observe that $\tilde{\varepsilon}$ is hence a modification $\tilde{\varepsilon}: h^{\prime} \Rightarrow h$. Now, the component $\rho_{(h, \varepsilon)}$ we have to define is a descent 2-morphism

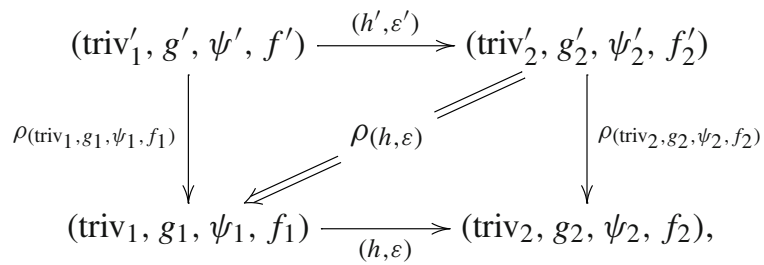

this is just a modification id $\circ h^{\prime} \Rightarrow h \circ$ id since the vertical arrows are the identity pseudonatural transformations. We define $\rho_{(h, \varepsilon)}$ from $\tilde{\varepsilon}$ and right and left unifiers in the obvious way. It is straightforward to see that this defines indeed a descent 2-morphism. Finally, we observe that the definitions $\rho_{(\operatorname{triv}, g, \psi, f)}$ and $\rho_{(h, \varepsilon)}$ furnish a pseudonatural equivalence as required.

The second part of the proof of Proposition 4.1.1 is the following lemma.

Lemma 4.1.3 There is a pseudonatural equivalence $\operatorname{id}_{\operatorname{Triv}_{\pi}^{2}(i)} \cong \operatorname{Rec}_{\pi} \circ \operatorname{Ex}_{\pi}$.

Proof For a 2-functor $F: \mathcal{P}_{2}(X) \rightarrow T$ and a $\pi$-local $i$-trivialization (triv, $t$ ), let (triv, $g, \psi, f$ ) be the associated descent data. We find a pseudonatural transformation

$$
\eta_{F}: F \rightarrow s^{*} R_{(\operatorname{triv}, g, \psi, f)}
$$

in the following way. Its component at a point $x \in X$ is the 1-morphism

$$
t(s(x)): F(x) \rightarrow \operatorname{triv}_{i}(s(x))
$$

in $T$. To define its component at a path $\gamma: x \rightarrow y$ we recall that $s(\gamma)$ is a composition of paths $\gamma_{i}: a_{i} \rightarrow b_{i}$ and jumps $\alpha_{i}$, so that we can compose $\eta_{F}(\gamma)$ from the pieces 

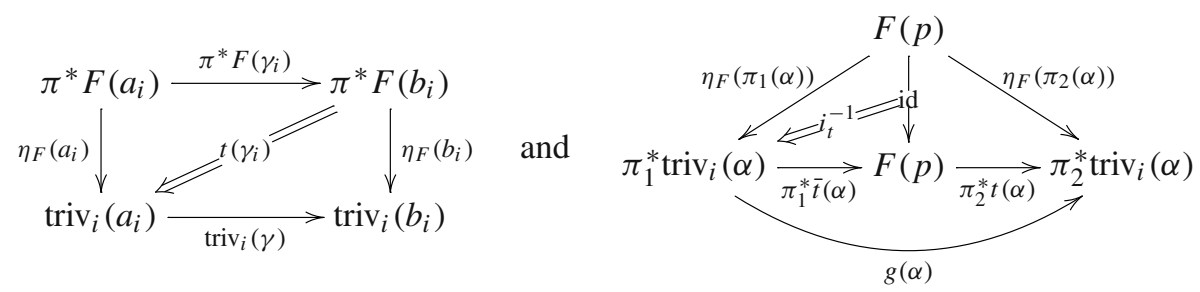

where $i_{t}: \bar{t} \circ t \Rightarrow$ id is the modification chosen to extract descent data. This defines the pseudonatural transformation $\eta_{F}$ associated to a 2-functor $F$.

Now let $A: F_{1} \rightarrow F_{2}$ be a pseudonatural transformation between two 2-functors with local trivializations $\left(\operatorname{triv}_{1}, t_{1}\right)$ and $\left(\operatorname{triv}_{2}, t_{2}\right)$. Let $(h, \varepsilon)$ the associated descent 1 -morphism. It is now straightforward to see that

$$
\eta_{A}:=i_{t_{1}}^{-1}: \eta_{F_{2}} \circ A \Rightarrow s^{*} R_{(h, \varepsilon)} \circ \eta_{F_{1}}
$$

defines a modification in such a way that both definitions together yield a pseudonatural transformation $\eta: \operatorname{id}_{\operatorname{Triv}_{\pi}^{2}(i)} \rightarrow \operatorname{Rec}_{\pi} \circ \mathrm{Ex}_{\pi}$. It is clear that $\eta$ is even a pseudonatural equivalence.

\subsection{Equivalence in the direct limit}

As mentioned in Sect. 2.1, path 2-groupoids come with 2-functors

$$
f_{*}: \mathcal{P}_{2}(M) \rightarrow \mathcal{P}_{2}(N)
$$

associated to smooth maps $f: M \rightarrow N$. In turn, these define 2-functors

$$
f^{*}: \operatorname{Funct}\left(\mathcal{P}_{2}(N), T\right) \rightarrow \operatorname{Funct}\left(\mathcal{P}_{2}(M), T\right) .
$$

The compatibility (2.1.1) of the 2-functors $f_{*}$ with the composition of smooth maps show that

$$
(g \circ f)^{*}=f^{*} \circ g^{*}
$$

for $g: N \rightarrow O$ another smooth map.

Now let $\pi_{1}: Y_{1} \rightarrow M$ and $\pi_{2}: Y_{2} \rightarrow M$ be surjective submersions and let $\xi: Y_{1} \rightarrow Y_{2}$ be a smooth map such that $\pi_{2} \circ \xi=\pi_{1}$. We call $\xi$ a refinement of $\pi_{2}$. Equation (4.2.1) implies that we obtain induced "restriction" 2-functors

$$
\operatorname{res}_{\xi}: \operatorname{Triv}_{\pi_{2}}^{2}(i) \rightarrow \operatorname{Triv}_{\pi_{1}}^{2}(i) \text { and } \operatorname{res}_{\xi}: \mathfrak{D e s}_{\pi_{2}}^{2}(i) \rightarrow \mathfrak{D e s}_{\pi_{1}}^{2}(i)
$$

and that these 2-functors themselves satisfy the compatibility condition (4.2.1), with respect to iterated refinements of surjective submersions.

In general, suppose that $S$ is a family of 2-categories parameterized by surjective submersions over a smooth manifold $M$. That is, if $\pi: Y \rightarrow M$ is a surjective 
submersion, then $S(\pi)$ is a 2-category. Suppose further that $F$ is a family of "refinement" 2 -functors parameterized by refinements of surjective submersions. That is, if $\zeta: Y^{\prime} \rightarrow Y$ is a refinement of a surjective submersion $\pi: Y \rightarrow M$ by $\pi^{\prime}: Y^{\prime} \rightarrow M$, then $F(\zeta): S(\pi) \rightarrow S\left(\pi^{\prime}\right)$ is a 2 -functor. Further, we require that $F\left(\zeta^{\prime} \circ \zeta\right)=F\left(\zeta^{\prime}\right) \circ F(\zeta)$ for iterated refinements. In this situation, one can form the direct limit 2-category

$$
S_{M}:=\lim _{\vec{\pi}} S(\pi) .
$$

We shall briefly describe a concrete model for this 2-category, the so-called Grothendieck construction. The precise form can be deduced from the general colimit description in $\infty$-categories; see [2, Corollary3.3.4.6]. An object of $S_{M}$ is a pair $(\pi, X)$ consisting of a surjective submersion $\pi: Y \rightarrow M$ and an object $X$ in $S(\pi)$. A common refinement of surjective submersions $\pi_{1}, \pi_{2}$ is a commutative diagram

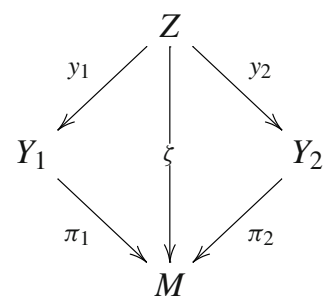

in which all maps are surjective submersions. A 1-morphism between objects $\left(\pi_{1}, X_{1}\right)$ and $\left(\pi_{2}, X_{2}\right)$ is a common refinement $\zeta$ together with a 1-morphism

$$
f: F\left(y_{1}\right)\left(X_{1}\right) \rightarrow F\left(y_{2}\right)\left(X_{2}\right)
$$

in $S(\zeta)$. The composition of two 1-morphisms

$$
\left(\zeta_{12}, f_{12}\right):\left(\pi_{1}, X_{1}\right) \rightarrow\left(\pi_{2}, X_{2}\right) \text { and }\left(\zeta_{23}, f_{23}\right):\left(\pi_{2}, X_{2}\right) \rightarrow\left(\pi_{3}, X_{3}\right)
$$

is defined as follows. We consider the fibre product $Z_{13}:=Z_{12} \times_{Y_{2}} Z_{23}$ as a common refinement $\zeta_{13}: Z_{13} \rightarrow M$ of $\pi_{1}$ and $\pi_{3}$. Then, we set

$$
\left(\zeta_{23}, f_{23}\right) \circ\left(\zeta_{12}, f_{12}\right):=\left(\zeta_{13}, F\left(\mathrm{pr}_{Z_{12}}\right)\left(f_{23}\right) \circ F\left(\mathrm{pr}_{Z_{23}}\right)\left(\zeta_{12}\right)\right)
$$

In order to define 2-morphisms between 1-morphisms $(\zeta, f)$ and $\left(\zeta^{\prime}, f^{\prime}\right)$ we consider pairs $(\omega, \alpha)$ of a common refinement $\omega: W \rightarrow M$ of $\zeta$ and $\zeta^{\prime}$ together with a 2-morphism

$$
\alpha: F(z)(f) \Rightarrow F\left(z^{\prime}\right)\left(f^{\prime}\right)
$$

in $S(\omega)$, where $z: W \rightarrow Z$ and $z^{\prime}: W \rightarrow Z^{\prime}$ are the two refinement maps. A 2 -morphism is then an equivalence class of pairs $(\omega, \alpha)$, where two pairs $\left(\omega_{1}, \alpha_{1}\right)$ and $\left(\omega_{2}, \alpha_{2}\right)$ are identified if the 2-morphisms agree when pulled back to the fibre product $W_{1} \times{ }_{Z \times{ }_{M}} Z^{\prime} W_{2}$. 
In the present situation, we form the direct limits

$$
\operatorname{Triv}^{2}(i)_{M}:=\lim _{\vec{\pi}} \operatorname{Triv}_{\pi}^{2}(i) \quad \text { and } \quad \mathfrak{D e s}^{2}(i)_{M}:=\lim _{\vec{\pi}} \mathfrak{D e s}_{\pi}^{2}(i)
$$

One checks by inspection that the 2-functor $\mathrm{Ex}_{\pi}$ commutes with the restriction 2functors $\operatorname{rec}_{\xi}$ of (4.2.2), so that the it induces a 2 -functor

$$
\operatorname{Ex}: \operatorname{Triv}^{2}(i)_{M} \rightarrow \mathfrak{D e s}^{2}(i)_{M}
$$

Since limits preserve equivalences, we conclude from Proposition 4.1.1:

Proposition 4.2.1 The 2-functor (4.2.3) is an equivalence of 2-categories.

Now we look at the full sub-2-category $\operatorname{Funct}_{i}\left(\mathcal{P}_{2}(M), T\right)$ of $\operatorname{Funct}\left(\mathcal{P}_{2}(M), T\right)$ over those 2-functors $F: \mathcal{P}_{2}(M) \rightarrow T$ that admit a $\pi$-local $i$-trivialization, for some surjective submersion $\pi: Y \rightarrow M$. We have a 2-functor

$$
v: \operatorname{Triv}^{2}(i)_{M} \rightarrow \operatorname{Funct}_{i}\left(\mathcal{P}_{2}(M), T\right)
$$

which simply forgets the local trivialization which is attached to the objects on the left hand side. The 2-functor $v$ is obviously an equivalence of 2-categories, since it is essentially surjective and the identity on Hom-categories. Summarizing, we get:

Theorem 4.2.2 There is an equivalence

$$
\operatorname{Des}^{2}(i)_{M} \cong \operatorname{Funct}_{i}\left(\mathcal{P}_{2}(M), T\right)
$$

between the 2-category of descent data and the 2-category of locally $i$-trivializable 2-functors, realized by a span of equivalences of 2-categories.

Theorem 4.2.2 is the main result of this article. In [6] we restrict it to an equivalence between important sub-2-categories: the one of smooth descent data (on the left hand side), and the one of transport 2-functors (on the right hand side). Transport 2-functors are an axiomatic formulation of connections on non-abelian gerbes.

Acknowledgements We would like to thank John Baez for many discussions and suggestions. We thank the Hausdorff Research Institute for Mathematics in Bonn for kind hospitality and support.

\section{Appendix A: Basic 2-category theory}

We introduce notions and facts that we need in this article. For a more complete introduction to 2-categories, see e.g. [1].

Definition A.1 A (small) 2-category consists of a set of objects, for each pair $(X, Y)$ of objects a set of 1-morphisms denoted $f: X \rightarrow Y$ and for each pair $(f, g)$ of 1-morphisms $f, g: X \rightarrow Y$ a set of 2-morphisms denoted $\varphi: f \Rightarrow g$, together with the following structure: 
1. For every pair $(f, g)$ of 1-morphisms $f: X \rightarrow Y$ and $g: Y \rightarrow Z$, a 1-morphism $g \circ f: X \rightarrow Y$, called the composition of $f$ and $g$.

2. For every triple $(f, g, h)$ of 1-morphisms $f: W \rightarrow X, g: X \rightarrow Y$ and $h: Y \rightarrow Z$, a 2-morphism

$$
a_{f, g, h}:(h \circ g) \circ f \Rightarrow h \circ(g \circ f)
$$

called the associator of $f, g$ and $h$.

3. For every object $X$, a 1-morphism id $X: X \rightarrow X$, called the identity 1-morphism of $X$.

4. For every 1-morphism $f: X \rightarrow Y, 2$-morphisms $l_{f}: f \circ \operatorname{id}_{X} \Rightarrow f$ and $r_{f}: \operatorname{id}_{Y} \circ f \Rightarrow f$, called the left and the right unifier.

5. For every pair $(\varphi, \psi)$ of 2-morphisms $\varphi: f \Rightarrow g$ and $\psi: g \Rightarrow h$, a 2-morphism $\psi \bullet \varphi: f \Rightarrow h$, called the vertical composition of $\varphi$ and $\psi$.

6. For every 1-morphism $f$, a 2-morphism id $f: f \Rightarrow f$, called the identity 2morphism of $f$.

7. For every triple $(X, Y, Z)$ of objects, 1-morphisms $f, f^{\prime}: X \rightarrow Y$ and $g, g^{\prime}: Y \rightarrow Z$, and every pair $(\varphi, \psi)$ of 2-morphisms $\varphi: f \Rightarrow f^{\prime}$ and $\psi: g \Rightarrow g^{\prime}$, a 2-morphism $\psi \circ \varphi: g \circ f \Rightarrow g^{\prime} \circ f^{\prime}$, called the horizontal composition of $\varphi$ and $\psi$.

This structure has to satisfy the following list of axioms:

(C1) The vertical composition of 2-morphisms is associative,

$$
(\phi \bullet \varphi) \bullet \psi=\phi \bullet(\varphi \bullet \psi)
$$

whenever these compositions are well-defined, while the horizontal composition is compatible with the associator in the sense that the diagram

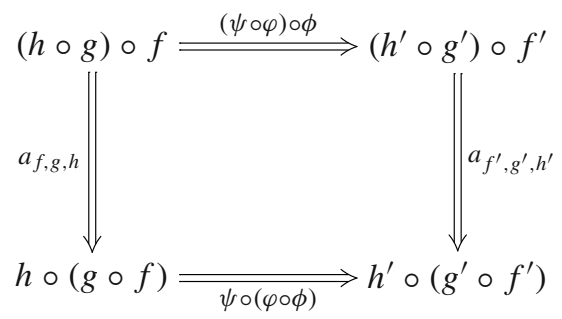

is commutative.

(C2) The identity 2-morphisms are units with respect to vertical composition,

$$
\varphi \bullet \operatorname{id}_{f}=\operatorname{id}_{g} \bullet \varphi
$$


for every 2-morphism $\varphi: f \Rightarrow g$, while the identity 1-morphisms are compatible with the unifiers and the associator in the sense that the diagram

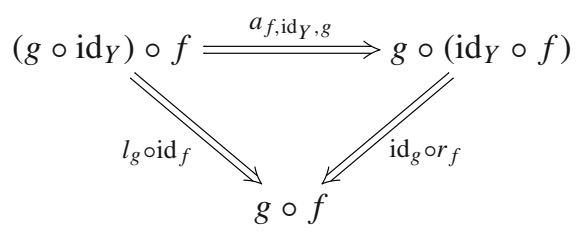

is commutative. Horizontal composition preserves the identity 2-morphisms in the sense that

$$
\operatorname{id}_{g} \circ \operatorname{id}_{f}=\operatorname{id}_{g \circ f} .
$$

(C3) Horizontal and vertical compositions are compatible in the sense that

$$
\left(\psi_{1} \bullet \psi_{2}\right) \circ\left(\varphi_{1} \bullet \varphi_{2}\right)=\left(\psi_{1} \circ \varphi_{1}\right) \bullet\left(\psi_{2} \circ \varphi_{2}\right)
$$

whenever these compositions are well-defined.

(C4) All associators and unifiers are invertible 2-morphisms and natural in $f, g$ and $h$, and the associator satisfies the pentagon axiom

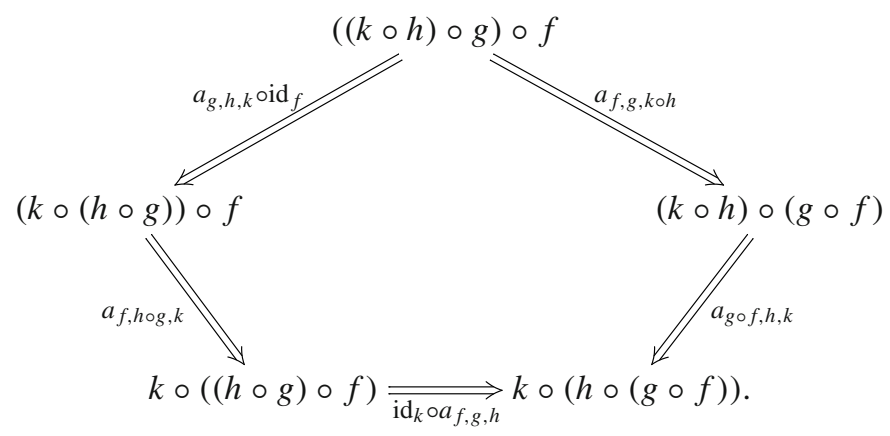

In (C4) we have called a 2-morphism $\varphi: f \Rightarrow g$ invertible or 2-isomorphism, if there exists a 2-morphism $\psi: g \Rightarrow f$ such that $\psi \bullet \varphi=\operatorname{id}_{f}$ and $\varphi \bullet \psi=$ $\mathrm{id}_{g}$. The axioms imply a coherence theorem: all diagrams of 2-morphisms whose arrows are labelled by associators, right or left unifiers, and identity 2-morphisms, are commutative. A 2-category is called strict, if

$$
(h \circ g) \circ f=h \circ(g \circ f) \quad \text { and } \quad a_{f, g, h}=\mathrm{id}_{h \circ g \circ f}
$$

for all triples $(f, g, h)$ of composable 1-morphisms, and if

$$
f \circ \mathrm{id}_{X}=f=\operatorname{id}_{Y} \circ f \quad \text { and } \quad r_{f}=l_{f}=\mathrm{id}_{f}
$$

for all 1-morphisms $f$. Strict 2-categories allow us to draw pasting diagrams, since multiple compositions of 1-morphisms are well-defined without putting brackets. Past- 
ing diagrams are often more instructive than commutative diagrams of 2-morphisms. For an explicit discussion of the strict case the reader is referred to Appendix A.1 in $[5]$.

Example A.2 Let $\mathfrak{C}$ be a monoidal category, i.e. a category equipped with a functor $\otimes: \mathfrak{C} \times \mathfrak{C} \rightarrow \mathfrak{C}$, a distinguished object $\mathbf{I}$ in $\mathfrak{C}$, a natural transformation $\alpha$ with components

$$
\alpha_{X, Y, Z}:(X \otimes Y) \otimes Z \rightarrow X \otimes(Y \otimes Z),
$$

and natural transformations $\rho$ and $\lambda$ with components

$$
\rho_{X}: \mathbf{I} \otimes X \rightarrow X \quad \text { and } \quad \lambda_{X}: X \otimes \mathbf{I} \rightarrow X
$$

which are subject to the usual coherence conditions, see, e.g. [3]. The monoidal category $\mathfrak{C}$ defines a 2 -category $\mathcal{B C}$ in the following way: it has a single object, the 1-morphisms are the objects of $\mathfrak{C}$ and the 2-morphisms between two 1-morphisms $X$ and $Y$ are the morphisms $f: X \rightarrow Y$ in $\mathfrak{C}$. The composition of 1-morphisms and the horizontal composition is the tensor product $\otimes$, and the associator $a_{X, Y, Z}$ is given by the component $\alpha_{Z, Y, X}$. The identity 1-morphism is the tensor unit $\mathbf{I}$, and the unifiers are given by the natural transformations $\rho$ and $\lambda$. The vertical composition and the identity are just the ones of $\mathfrak{C}$. It is straightforward to check that axioms (C1) to $(\mathrm{C} 4)$ are either satisfies due to the axioms of the category $\mathfrak{C}$, the functor $\otimes$, or the natural transformations $\alpha, \rho$ and $\lambda$, or due to the coherence axioms. The 2-category $\mathcal{B C}$ is strict if and only if the monoidal category $\mathfrak{C}$ is strict.

In any 2-category, a 1-morphism $f: X \rightarrow Y$ is called invertible, if there exists another 1-morphism $g: Y \rightarrow X$ together with natural 2-isomorphisms $i: g \circ f \Rightarrow \operatorname{id}_{X}$ and $j: \operatorname{id}_{Y} \Rightarrow f \circ g$ such that the diagrams
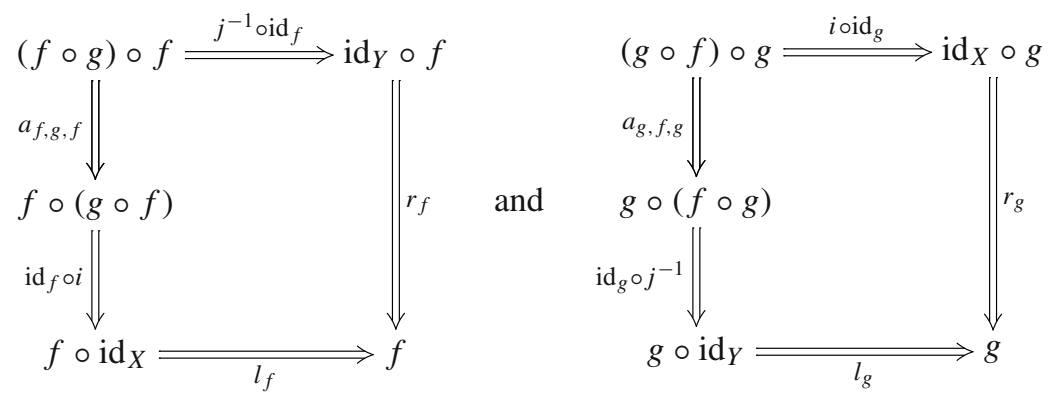

are commutative. Let us remark that neither in the strict nor in the general case the inverse 1-morphism $g$ is uniquely determined. We call a triple $(g, i, j)$ a weak inverse of $f$. By 1-isomorphism we mean an invertible 1-morphism together with a weak inverse.

Remark A.3 Often a 2-category is called bicategory, while a strict 2-category is called 2-category. Invertible 1-morphisms are often called adjoint equivalences. 
Definition A.4 A (strict) 2-category in which every 1-morphism and every 2morphism is invertible, is called (strict) 2-groupoid.

The following definition generalizes the one of a functor between categories.

Definition A.5 Let $S$ and $T$ be two 2-categories. A 2-functor $F: S \rightarrow T$ assigns

1. an object $F(X)$ in $T$ to each object $X$ in $S$,

2. a 1-morphism $F(f): F(X) \rightarrow F(Y)$ in $T$ to each 1-morphism $f: X \rightarrow Y$ in $S$, and

3. a 2-morphism $F(\varphi): F(f) \Rightarrow F(g)$ in $T$ to each 2-morphism $\varphi: f \Rightarrow g$ in $S$.

Furthermore, it has

(a) A 2-isomorphism $u_{X}: F\left(\operatorname{id}_{X}\right) \Rightarrow \operatorname{id}_{F(X)}$ in $T$ for each object $X$ in $S$, and

(b) A 2-isomorphism $c_{f, g}: F(g) \circ F(f) \Rightarrow F(g \circ f)$ in $T$ for each pair of composable 1-morphisms $f$ and $g$ in $\mathrm{S}$.

Four axioms have to be satisfied:

(F1) The vertical composition is respected in the sense that

$$
F(\psi \bullet \varphi)=F(\psi) \bullet F(\varphi) \quad \text { and } \quad F\left(\operatorname{id}_{f}\right)=\operatorname{id}_{F(f)}
$$

for all composable 2-morphisms $\varphi$ and $\psi$, and any 1-morphism $f$.

(F2) The horizontal composition is respected in the sense that the diagram

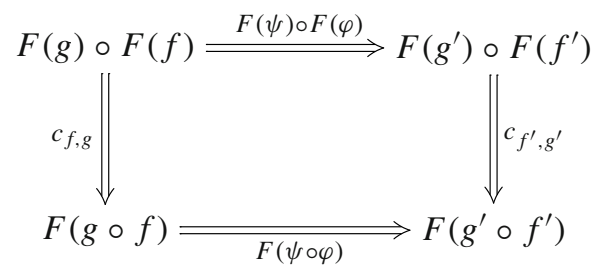

is commutative for all horizontally composable 2-morphisms $\varphi$ and $\psi$.

(F3) The compositor $c_{f, g}$ is compatible with the associators of $S$ and $T$ in the sense that the diagram

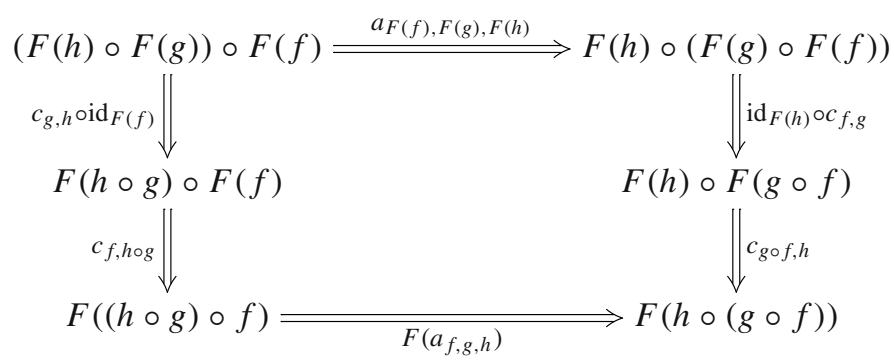

is commutative for all composable 1-morphisms $f, g$ and $h$. 
(F4) Compositor and unitor are compatible with the unifiers of $S$ and $T$ in the sense that the diagrams
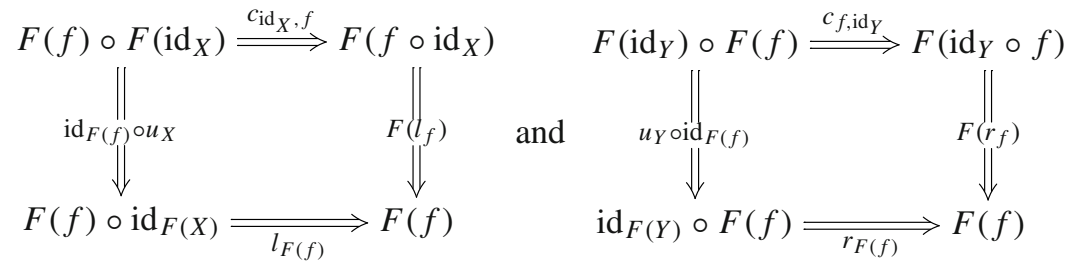

are commutative for every 1-morphism $f$.

Sometimes we represent a 2-functor $F: S \rightarrow T$ diagrammatically as an assignment

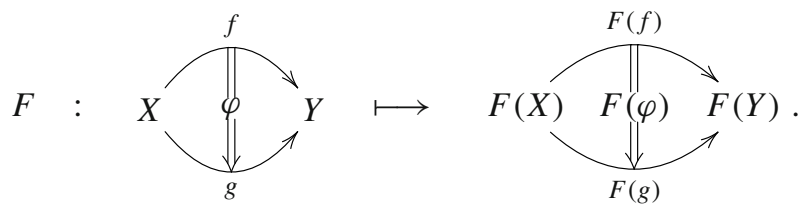

In case that the 2-category $T$ is strict, and the axioms (F2) to (F4) can be expressed by pasting diagrams in the following way:

- Axioms (F2) is equivalent to the equality

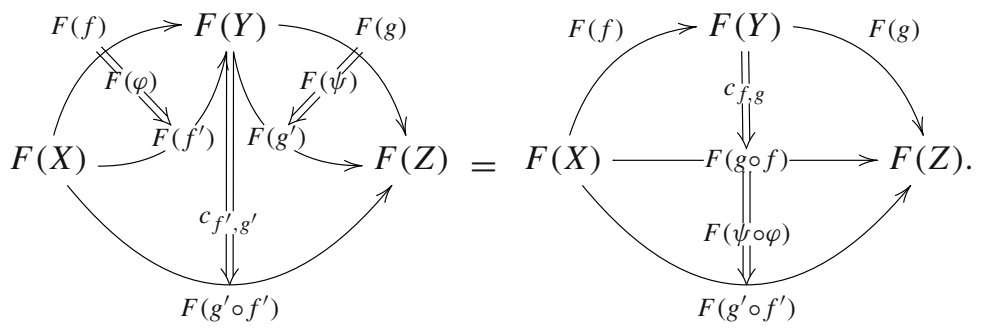

- Axiom (F3) is equivalent to the tetrahedron identity

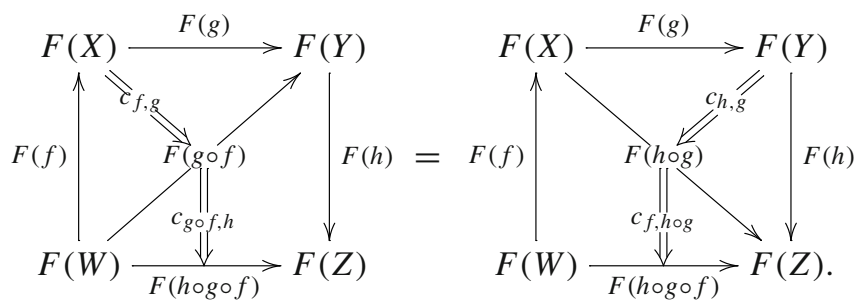

- Axiom (F4) is equivalent to the equalities

$$
c_{\mathrm{id}_{X}, f}=\operatorname{id}_{F(f)} \circ u_{X} \quad \text { and } \quad c_{f, \mathrm{id}_{Y}}=u_{Y} \circ \operatorname{id}_{F(f)} .
$$


We use two layers of strictness conditions for 2-functors, normally in a situation when $S$ and $T$ are strict 2-categories. Firstly, we call a 2-functor $F: S \rightarrow T$ normalized if

$$
F\left(\operatorname{id}_{X}\right)=\operatorname{id}_{F(X)} \quad \text { and } \quad u_{X}=\operatorname{id}_{i d_{F(X)}}
$$

for all objects $X$ in $S$, and if

$$
F(g) \circ F(f)=\mathrm{id}_{F(X)} \quad \text { and } \quad c_{f, g}=\mathrm{id}_{\mathrm{id}_{X}}
$$

for all 1-morphisms $f: X \rightarrow Y$ and $g: Y \rightarrow X$ such that $g \circ f=\operatorname{id}_{X}$. Roughly speaking, normalized 2-functors strictly respect identities and inverses. The second and stronger requirement is that the 2-functor $F: S \rightarrow T$ is strict: this requires A.2 while A. 3 is superseded by the condition that

$$
F(g) \circ F(f)=F(g \circ f) \quad \text { and } \quad c_{f, g}=\operatorname{id}_{F(g \circ f)}
$$

for all composable 1-morphisms $f$ and $g$. In case of strict 2-functors between strict 2-categories only axioms (F1) and (F2) remain, claiming that both compositions are respected.

The following definition generalizes a natural transformation between functors.

Definition A.6 Let $F_{1}$ and $F_{2}$ be two 2 -functors from $S$ to $T$. A pseudonatural transformation $\rho: F_{1} \rightarrow F_{2}$ assigns

1. A 1-morphism $\rho(X): F_{1}(X) \rightarrow F_{2}(X)$ in $T$ to each object $X$ in $S$, and

2. A 2-isomorphism $\rho(f): \rho(Y) \circ F_{1}(f) \Rightarrow F_{2}(f) \circ \rho(X)$ in $T$ to each 1-morphism $f: X \rightarrow Y$ in $S$,

such that two axioms are satisfied:

(T1) The composition of 1-morphisms in $S$ is respected in the sense that the diagram

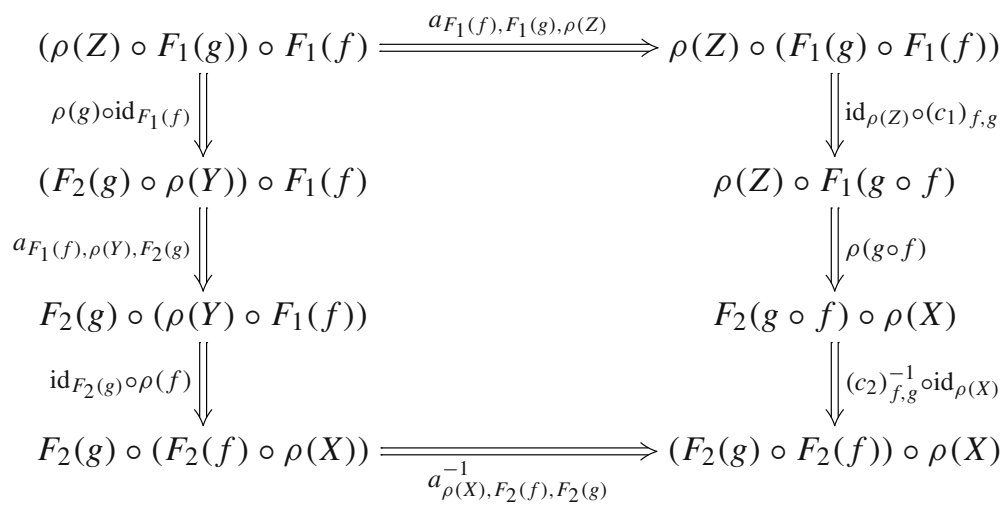

is commutative for all composable 1-morphisms $f$ and $g$. Here, $a$ is the associator of the 2-category $T$ and $c_{1}$ and $c_{2}$ are the compositors of the 2-functors $F_{1}$ and $F_{2}$, respectively. 
(T2) It is natural in the sense that the diagram

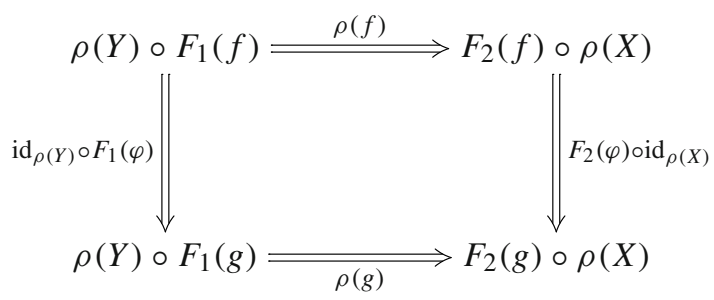

is commutative for all 2-morphisms $\varphi: f \Rightarrow g$.

If one considers a version of pseudonatural transformations where the 2-morphisms $\rho(f)$ do not have to be invertible, there is a third axiom related to the value of $\rho$ at the identity 1-morphism id in $_{X}$ of abject $X$ in $S$. In our setup this axiom is automatically satisfied, as the following lemma shows.

Lemma A.7 Let $\rho: F_{1} \rightarrow F_{2}$ be a pseudonatural transformation between 2functors $F_{1}$ and $F_{2}$ with unitors $u^{1}$ and $u^{2}$, respectively. Then, the following assertions hold.

(i) The diagram

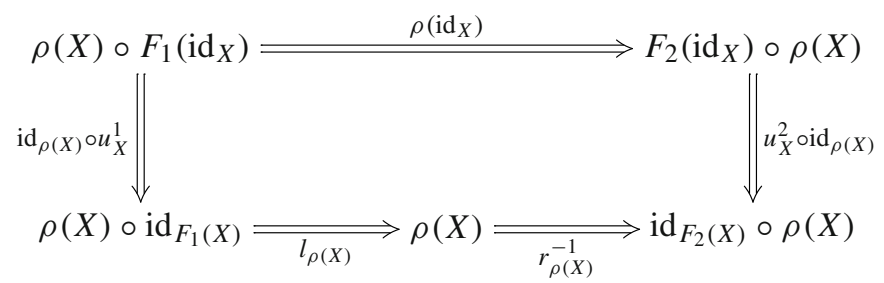

is commutative.

(ii) If $S$ and $T$ are strict 2-categories, and $F_{1}$ and $F_{2}$ are normalized 2-functors, then

$$
\rho\left(\operatorname{id}_{X}\right)=\operatorname{id}_{\rho(X) \circ F_{1}\left(\operatorname{id}_{X}\right)}=\operatorname{id}_{F_{2}\left(\operatorname{id}_{X}\right) \circ \rho(X)}
$$

for all objects $X$ in $S$, and

$$
\rho(g) \circ \mathrm{id}_{F_{1}(f)}=\operatorname{id}_{F_{2}(g)} \circ \rho(f)^{-1}
$$

for all 1-morphisms $f, g$ in $S$ with $g \circ f=\mathrm{id}$.

Proof For (i) one applies axiom (T1) to 1-morphisms $f=g=\mathrm{id}_{X}$. Then one uses axiom (T2) for $\rho$, axiom (F4) for both 2 -functors, axiom (C2) for $T$, and the invertibility of the 2-morphism $\rho(g)$ and of the 1-morphism $F_{2}\left(\mathrm{id}_{X}\right)$. The first claim of (ii) follows from (i) under the strictness assumptions, and the second claim follows from the first claim and axiom (T1). 
Sometimes we represent a pseudonatural transformation $\rho: F_{1} \rightarrow F_{2}$ diagrammatically by

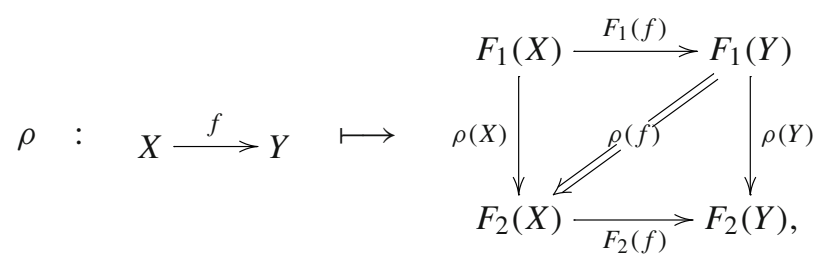

and if the 2-category $T$ is strict, the axioms can be expressed by pasting diagrams in the following way:

- Axiom (T1) is equivalent to

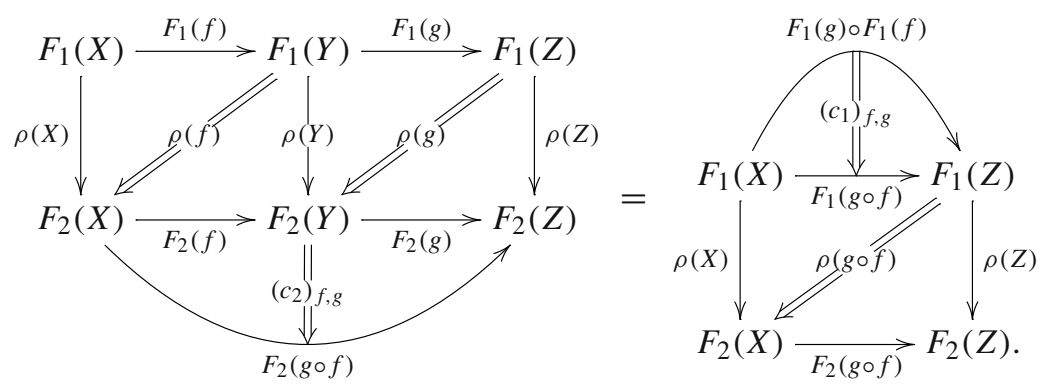

- Axiom (T2) is equivalent to

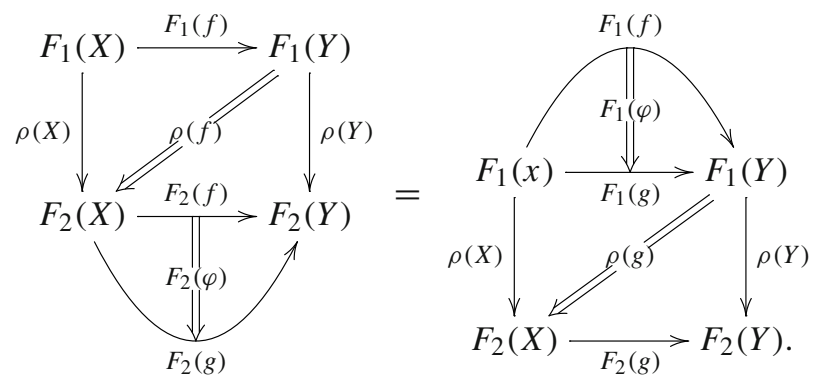

We need one more definition for situations where two pseudonatural transformations are present.

Definition A.8 Let $F_{1}, F_{2}: S \rightarrow T$ be two 2 -functors and let $\rho_{1}, \rho_{2}: F_{1} \rightarrow F_{2}$ be pseudonatural transformations. A modification $\mathcal{A}: \rho_{1} \Rightarrow \rho_{2}$ assigns a 2-morphism

$$
\mathcal{A}(X): \rho_{1}(X) \Rightarrow \rho_{2}(X)
$$


in $T$ to any object $X$ in $S$, such that the diagram

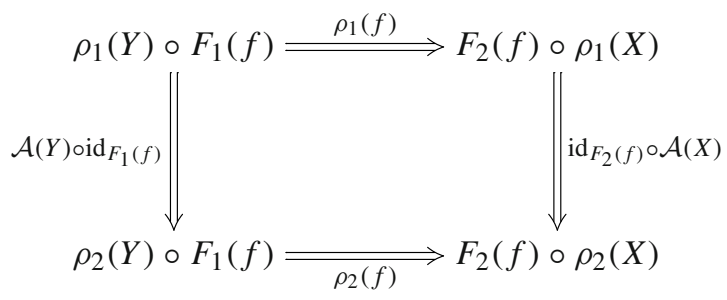

is commutative for every 1-morphism $f$ in $S$.

In the case that $T$ is a strict 2-category, the latter diagram is equivalent to a pasting diagram, see Definition A.4 in [5].

As one might expect, 2-functors, pseudonatural transformations, and modifications fit into the structure of a 2-category that we denote by Funct $(S, T)$. It is strict if and only if $T$ is strict. Note that the definition of invertibility in a 2-category applies; we call a 2-isomorphism in the 2-category $\operatorname{Funct}(S, T)$ invertible modification, and a 1-isomorphism pseudonatural equivalence. This leads to the following

Definition A.9 Let $S$ and $T$ be 2-categories. A 2-functor $F: S \rightarrow T$ is called an equivalence of 2-categories, if there exists a 2-functor $G: T \rightarrow S$ together with pseudonatural equivalences $\rho_{S}: G \circ F \rightarrow \operatorname{id}_{S}$ and $\rho_{T}: F \circ G \rightarrow \operatorname{id}_{T}$.

\section{References}

1. Leinster, T.: Basic bicategories. (Preprint)

2. Lurie, J.: Higher topos theory, Ann. Math. Stud., vol. 109. Princeton University Press, Princeton (2009)

3. Mac Lane, S.: Categories for the working mathematician, 2nd edn. Springer, Berlin (1997)

4. Schreiber, U., Waldorf, K.: Parallel transport and functors. J. Homotopy Relat. Struct. 4, 187-244 (2009)

5. Schreiber, U., Waldorf, K.: Smooth functors vs. differential forms. Homol. Homot. Appl. 13(1), 143-203 (2011)

6. Schreiber, U., Waldorf, K.: Connections on non-abelian gerbes and their holonomy. Theory Appl. Categ. 28(17), 476-540 (2013) 